\title{
WestVirginiaUniversity
}

THE RESEARCH REPOSITORY @ WVU

Graduate Theses, Dissertations, and Problem Reports

2005

\section{Localized double-quantum filtered correlated spectroscopy on 3T MRI/MRS scanner}

\author{
Senthil Ramamurthy \\ West Virginia University
}

Follow this and additional works at: https://researchrepository.wvu.edu/etd

\section{Recommended Citation}

Ramamurthy, Senthil, "Localized double-quantum filtered correlated spectroscopy on 3T MRI/MRS scanner" (2005). Graduate Theses, Dissertations, and Problem Reports. 1882.

https://researchrepository.wvu.edu/etd/1882

This Thesis is protected by copyright and/or related rights. It has been brought to you by the The Research Repository @ WVU with permission from the rights-holder(s). You are free to use this Thesis in any way that is permitted by the copyright and related rights legislation that applies to your use. For other uses you must obtain permission from the rights-holder(s) directly, unless additional rights are indicated by a Creative Commons license in the record and/ or on the work itself. This Thesis has been accepted for inclusion in WVU Graduate Theses, Dissertations, and Problem Reports collection by an authorized administrator of The Research Repository @ WVU. For more information, please contact researchrepository@mail.wvu.edu. 


\title{
Localized Double-Quantum Filtered Correlated Spectroscopy on 3T MRI/MRS Scanner
}

\author{
by \\ Senthil Ramamurthy \\ Thesis submitted to the \\ College of Engineering and Mineral Resources \\ at West Virginia University \\ in partial fulfillment of the requirements \\ for the degree of \\ Master of Science \\ in \\ Electrical Engineering \\ Dr. Matthew C. Valenti, Chair \\ Dr. Wils L. Cooley \\ Dr. Mark A. Jerabek \\ Dr. Raymond R. Raylman \\ Dr. Sendhil S. Velan
}

Lane Department of Computer Science and Electrical Engineering

Morgantown, West Virginia

2005

Keywords: Multiple-Quantum Filter, Double-Quantum Filter, COSY

Copyright 2005 Senthil Ramamurthy 


\author{
Abstract \\ Localized Double-Quantum Filtered Correlated Spectroscopy on 3T MRI/MRS \\ Scanner \\ by \\ Senthil Ramamurthy \\ Master of Science in Electrical Engineering \\ West Virginia University \\ Dr. Matthew C. Valenti, Chair
}

Standard localized magnetic resonance spectroscopic sequences employ single quantum coherences for excitation and detection. This results in a complex spectra containing large number of resonances. In this thesis we have developed a novel volume localized spectroscopic technique that excites multiple quantum coherences in coupled spin clusters (metabolites) and then reconvert them into single quantum coherences for detection. This results in filtering of uncoupled resonances and also suppresses water with high efficiency. The resulting spectra is reduced in complexity due to the absence of uncoupled resonances.

The technique has been developed in volume localized mode and implemented on a $3 \mathrm{~T}$ scanner. The technique has been designed to get single-scan coherence pathway selection under gradient-controlled echo filtering, ensuring optimum use of the dynamic range of the receiver electronics.

The multiple quantum preparation sandwich consists of $90^{\circ}-t_{1}-90^{\circ} \mathrm{RF}$ pulse sequence. The period $t_{1}$ can be optimized for maximizing the desired multiple quantum coherence. The final $90^{\circ}$ pulse reconverts the multiple quantum coherences into single quantum coherences. The slice selection gradients are combined with the three RF pulses for volume localization.

The sequence has been tested on phantoms of ethanol and ethyl acetate. The singlets are suppressed as expected. Only the double quantum coherence are able to pass through the double quantum coherence pathway. Preliminary in vivo results for identification of EMCL and IMCL in human calf muscle has been presented. 


\section{Acknowledgments}

My sincere thanks to Dr. Sendhil Velan, for introducing me to the interesting world of NMR, being a source of inspiration, and for his untiring efforts. I would like to thank to Dr. Matthew C. Valenti for excellent advising and helping me a lot in this project. I would also like to extend my sincere thanks to my committee members Dr. Mark A. Jerabek, Dr. Wils L. Cooley and Dr. Raymond R. Raylman for their help and excellent suggestions through out the project.

I would like to thank my friends Niveditha, Balaji, Arvind and Sukanya for making my stay at Morgantown to be cherished for years to come. Without their constant support and encouragement this project wouldnt have been fruitful. Last but not the least, I would like to thank my brother Balu, for his care, affection and constant encouragement through out my Masters degree. I would like to thank my parents, for everything they have provided me. Without them, my degree would have just been a dream. 


\section{Contents}

Acknowledgments $\quad$ iii

List of Figures vii

List of Tables $\quad$ ix

1 Introduction 1

2 One and Two Dimensional MRS 5

2.1 Magnetic Properties of the Nuclei . . . . . . . . . . . . . 5

2.1.1 Interaction of Magnetic Moment with Magnetic Field . . . . . 5

2.1.2 Precession . . . . . . . . . . . . . . . . 6

2.1.3 Boltzmann Distribution . . . . . . . . . . . . . 7

2.1.4 The Rotating Frame of Reference . . . . . . . . . . . . . . . 7

2.2 One Dimensional MRS . . . . . . . . . . . . . . . . . . . 8

2.2 .1 Chemical Shifts . . . . . . . . . . . . . . . . 9

2.2.2 Spin-Spin Coupling . . . . . . . . . . . . . . . . . . 11

2.3 Energy Level of a Two Spin System . . . . . . . . . . . . . . . . . . 11

2.3.1 Energy Levels . . . . . . . . . . . . . . . . . . . . 11

2.3.2 Two Spin System . . . . . . . . . . . . . . . . . . . . . . . 13

2.3.3 Energy Level Populations . . . . . . . . . . . . . . . . 15

2.4 Introduction to $2 \mathrm{D}$ MRS . . . . . . . . . . . . . . 16

3 Clinical MRS $\quad 18$

3.1 Introduction to Clinical MR System . . . . . . . . . . . . . . . 18

3.1 .1 Magnet System . . . . . . . . . . . . . . . . . . . . . 19

3.1.2 Magnetic and RF Shielding . . . . . . . . . . . . . . . . 20

3.1 .3 Shimming . . . . . . . . . . . . . . . . 20

3.1 .4 Gradient System . . . . . . . . . . . . . . . . 20

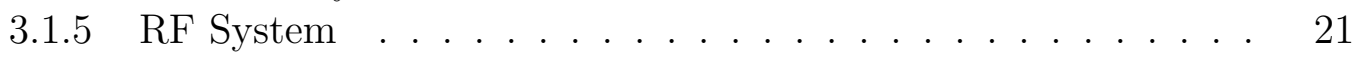

3.1.6 Patient Table ..................... . . . . 21

3.2 RF Pulses . . . . . . . . . . . . . . . . . . . . . . . 21

3.3 RF Coils . . . . . . . . . . . . . . . . . . . . . . . 22

3.4 Volume Localized MRS . . . . . . . . . . . . . . . . . . . . . . . . . . . . . . . . . 23

3.4.1 Need for Localization . . . . . . . . . . . . . . . . 23 
3.4 .2 Water Suppression . . . . . . . . . . . . . . . . 23

3.4.3 Outer Volume Suppression . . . . . . . . . . . . . . . . . . . 24

3.5 Single Voxel Localization . . . . . . . . . . . . . . . . . . . . . 25

3.5.1 Image Selected In Vivo Spectroscopy (ISIS) . . . . . . . . . . 25

3.5.2 STimulated Echo Acquisition Mode (STEAM) . . . . . . . . 26

3.5.3 Point RESolved Spectroscopy (PRESS) . . . . . . . . . . . . . 28

3.6 Limitations of $1 \mathrm{D}$ MRS . . . . . . . . . . . . . . . . . . . 29

3.7 Localized 2D MRS . . . . . . . . . . . . . . . . . . . . . . 29

3.7.1 Interpreting the 2D Spectrum . . . . . . . . . . . . . 31

4 Multiple Quantum Coherences 33

4.1 Product Operator Formalism . . . . . . . . . . . . . . . . . . . 34

4.1.1 Spin Populations . . . . . . . . . . . . . . . . . . . . . . . . . . . . 35

4.1 .2 Magnetization . . . . . . . . . . . . . . . . . 36

4.1.3 Coherence . . . . . . . . . . . . . . . . . . 36

4.1.4 Evolution . . . . . . . . . . . . . . . . . 38

4.2 Multiple Quantum Coherence(MQC) . . . . . . . . . . . . . . 38

4.3 Creating Multiple Quantum Coherence(MQC) . . . . . . . . . . . . . 39

4.3.1 $\left(\frac{\pi}{2}\right)_{\phi}-\frac{\tau}{2}-(\pi)_{\phi}-\frac{\tau}{2}-\left(\frac{\pi}{2}\right)_{\phi+\psi}$ excitation . . . . . . . . . . 39

$4.3 .2 \quad\left(\frac{\pi}{2}\right)_{x}-\tau-\left(\frac{\pi}{2}\right)_{x}$ excitation . . . . . . . . . . . 39

4.4 Detection of Multiple Quantum Coherences . . . . . . . . . . . . . . 40

4.4.1 Single Point Acquisition . . . . . . . . . . . . . . . . 41

4.4.2 Multiple Point Acquisition . . . . . . . . . . . . . . . . . . 41

4.5 Special Properties of Multiple Quantum Coherences . . . . . . . . . . 42

4.6 Order Selective Detection . . . . . . . . . . . . . . . . 43

4.7 Coherence Transfer Pathways . . . . . . . . . . . . . . . . . . . 43

5 Development of Localized DQF-COSY $\mathbf{4 5}$

5.1 Motivation for development of the DQF Localized MRS . . . . . . . . 45

5.2 Design of the Localized DQF Sequence . . . . . . . . . . . . . . . 46

5.3 Theory of Localized DQF . . . . . . . . . . . . . . . . . 46

5.4 Environment for Pulse Programming

in $\mathrm{C}(\mathrm{EPIC}) \ldots \ldots \ldots \ldots$. . . . . . . . . . . . . . . . . . . . . 48

5.4.1 Interprocess Communication in EPIC . . . . . . . . . . . 49

5.5 Implementing the Sequence in EPIC . . . . . . . . . . . . . 50

6 Experimental Results $\quad \mathbf{5 2}$

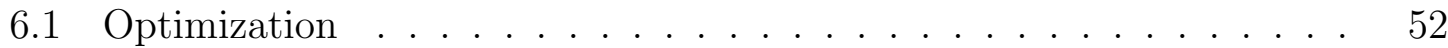

6.1.1 Optimizing Water Suppression . . . . . . . . . . . . 52

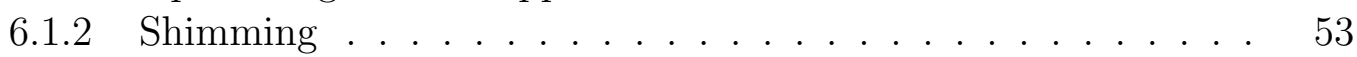

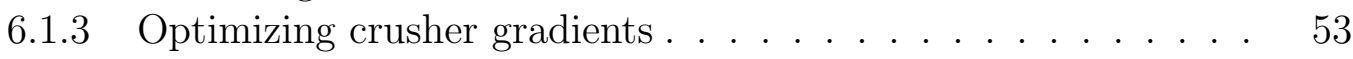

6.2 Scan . . . . . . . . . . . . . . . . . 53

6.3 Post Processing Data . . . . . . . . . . . . . . . . . 54

6.3.1 Apodization . . . . . . . . . . . . . . 54

6.3 .2 Linear Prediction . . . . . . . . . . . . . . . . 55 
6.4 Results and Discussion . . . . . . . . . . . . . . . 55

6.4.1 Results on Ethanol Phantom . . . . . . . . . . . . . 55

6.4.2 Results on Ethyl Acetate . . . . . . . . . . . . . . . . . . 58

6.4.3 In Vivo Results on Human Calf Muscle . . . . . . . . . . . . . 59

7 Summary, Conclusion and Future Research 63

$\begin{array}{ll}\text { References } & 65\end{array}$ 


\section{List of Figures}

2.1 Spin Orientations . . . . . . . . . . . . . . . . 6

2.2 Precession Magnetic Moment . . . . . . . . . . . . . . . . . 7

2.3 energy levels . . . . . . . . . . . . . . . . . . . . . 8

2.4 rotating frame of reference . . . . . . . . . . . . . . . 9

2.5 Shielding Effect . . . . . . . . . . . . . . . . . . 10

2.6 1D Spectrum of Ethanol showing chemical shifts and spin-spin coupling 12

2.7 Energy level diagram of a coupled two spin system . . . . . . . . . . 14

2.8 Boltzmann distribution of spins . . . . . . . . . . . . . 16

3.1 Water suppression in GE 3.0T Signa Horizon LX Scanner. Reproduced

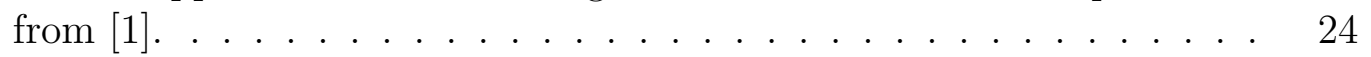

3.2 Outer volume suppression in GE 3.0T Signa Horizon LX Scanner. Re-

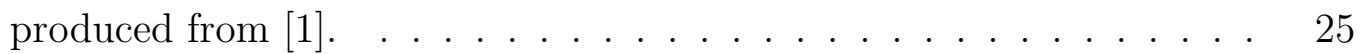

3.3 Image-selected in vivo spectroscopy (ISIS). Reproduced from $\lfloor 2\rfloor$. . . 26

3.4 STEAM pulse sequence. Reproduced from $[2] \ldots \ldots$. . . . . . . . . 27

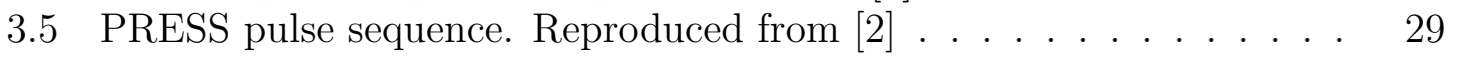

3.6 Simulated spectrum of various metabolites at 3.0T . . . . . . . . . . 30

3.7 Schematic COSY spectrum . . . . . . . . . . . . . . . . . 31

4.1 Energy level diagram of a coupled two spin system . . . . . . . . . 33

4.2 Three pulse sandwich for excitation of multiple quantum coherences 40

4.3 Two pulse sandwich for excitation of multiple quantum coherences . 40

5.1 Interprocess Communication in EPIC. Reproduced from [1] . . . . . 49

6.1 Chemical Structure of Ethanol . . . . . . . . . . . . . . . 56

6.2 One dimensional spectrum of Ethanol Phantom . . . . . . . . . . 56

6.3 L-COSY spectrum of Ethanol Phantom . . . . . . . . . . . . . . 57

6.4 DQF-COSY spectrum of Ethanol Phantom . . . . . . . . . . . . 57

6.5 Chemical structure of ethyl acetate . . . . . . . . . . . . 58

6.6 One-dimensional spectrum of ethyl acetate . . . . . . . . . . . . 59

6.7 L-COSY spectrum of ethyl acetate . . . . . . . . . . . . . . 59

6.8 DQF-COSY spectrum of ethyl acetate with a lower threshold . . . . . 60

6.9 Calf muscle image . . . . . . . . . . . . . . . . . . . 60

6.10 One-dimensional spectrum of the voxel on human calf muscle . . . . . 61 
6.11 DQF-COSY spectrum of the voxel on human calf muscle . . . . . . . 62 


\section{List of Tables}

5.1 Anatomical to logical-co-ordinate conversion table . . . . . . . . . 50

5.2 Gradient ratios for coherence pathway selection . . . . . . . . . . 51 


\section{Chapter 1}

\section{Introduction}

The field of spectroscopy is concerned with the interaction between matter and electromagnetic radiation. Atoms and molecules have a range of discrete energy levels corresponding to different electronic, vibrational or rotational states. The interaction between atoms and electromagnetic radiation is characterized by the absorption and emission of photons by the atoms, such that the energy of the photon exactly matches an energy level difference in the atom. Since the energy of a photon is proportional to its frequency, the different forms of spectroscopy are distinguished on the basis of the frequencies involved. Nuclear Magnetic Resonance (NMR) spectroscopy uses radio frequencies $(\mathrm{RF})$ which are typically in the range of few hundred $M H z$.

NMR is the study of the magnetic properties of nuclei. The absorption and emission of electromagnetic radiation can be observed when the nuclei are placed in a strong external magnetic field. NMR was discovered independently by Purcell, Torrey and Pound at MIT[3], and by Bloch, Hansen and Packard [4] at Stanford University in 1945. In 1952 Bloch and Purcell shared the Nobel Prize for Physics in recognition of their pioneering achievements $\mid 3,4,5,6\rfloor$. Proctor and $\mathrm{Yu}[7\rfloor$ and independently Dickinson[8] observed that nuclei within the same molecule can absorb energy at different resonant frequencies. These chemical-shift effects were directly related to the chemical environment of the nuclei. 
NMR spectra were recorded in the continuous wave mode in which the magnetic field strength or the radio frequency was swept through the spectral area of interest, while keeping the other fixed. In 1966, Ernst and Anderson $[9]$ introduced pulsed NMR in combination with Fourier transformation. Pulsed or Fourier transform NMR is the heart of all modern NMR experiments.

The induced energy level difference of nuclei in an external magnetic field is very small when compared to the thermal energy, making the energy levels almost equally populated. As a result, the absorption of photons is very low, making NMR an insensitive technique when compared to the other forms of spectroscopy. This low-energy absorption also makes NMR a non-invasive and non-destructive technique, ideally suited for in vivo measurements. Soon after the discovery of NMR, researchers used NMR to investigate living objects. In 1950, Shaw and Elsken [10] used proton NMR to investigate water. Odebald and Lindstrom [11] obtained proton spectrum from a number of mammalian preparations in 1955. In 1971 Damadian [12 | reported that NMR properties (relaxation times) of malignant tissues significantly differ from normal tissue, suggesting that proton NMR may have a diagnostic value.In the early 1970's, the first experiments of high-resolution NMR on intact living tissues, were reported. Moon and Richards $\mid 13\rfloor$ used ${ }^{31} \mathrm{P}$ NMR on intact red blood cells and showed that intracellular $\mathrm{pH}$ can be determined from chemical shift differences. In 1974, Hoult et al $[14\rfloor$ reported the first study of ${ }^{31} \mathrm{P}$ NMR to study intact excised rat hindleg muscle.

Current state-of-the-art one-dimensional techniques allows the acquisition of excellent water-suppressed spectra. Due to the recent improvements in the design of $B_{0}$ gradient and RF coils, ${ }^{1} \mathrm{H}$ MR spectra have been recorded in the human brain with excellent water suppression using short echo times, as short as $15 \mathrm{~ms}$, and several cerebral metabolites have been identified $[15,16,17]$. During the past decade alterations in several metabolites, namely, N-acetylaspartate (NAA), glutamate/glutamine (Glx), choline $(\mathrm{Ch})$, creatine $(\mathrm{Cr})$, myo-inositol $(\mathrm{mI})$, and $\gamma$-aminobutyrate (GABA) have been reported in different pathologic states involving the central nervous system 
(CNS). Absolute quantification of cerebral metabolites in vivo has also been reported for only a few metabolites, with limited success. However, due to the limited field strengths (1.5T and 3T) the signals from metabolites overlap severely and hamper their identification and quantification. Thus, the information derived from the MRS spectrum is only for a few well separated metabolites.

Several researchers have attempted to design and develop new pulse sequences that can separate the overlapping metabolites at clinical field strengths using approaches of one-dimensional spectral editing $\lfloor 18,19\rfloor$, multiple-quantum coherences $[20,21,22]$ and two-dimensional MRS $[23,24,25]$. One-dimensional (1D) MR spectral editing techniques to unravel the overlapping resonances rely on spin-spin coupled proton metabolites that have well-separated multiplets. Localized one-dimensional multiple-quantum coherence filter techniques, have been proposed to detect specific metabolites. Localized 2D MRS techniques like L-COSY $[26]$ has been suggested to detect all the metabolites. This results in a very complex spectrum, where it may be difficult to identify the cross peaks which are close to the diagonal peaks.

In this thesis we have developed a novel localized two-dimensional double-quantumfiltered correlated MRS pulse sequence (Localized DQF-COSY) on a GE 3T MRI/MRS scanner. This sequence reduces the complex spectrum by eliminating the uncoupled resonances (nuclear spins that do not exhibit scalar-coupling). This results in a much simpler spectrum, as compared to the L-COSY spectrum. The sequence was programmed in EPIC environment and tested for hardware and software issues.

The thesis is organized as follows. A literature survey of various $1 \mathrm{D}$ and $2 \mathrm{D}$ MRS techniques for detection of specific metabolites has been presented in chapter 1. Chapter 2 introduces the physics of magnetic resonance. The discussion includes magnetic property of nuclei, chemical shifts, couplings, energy levels and Boltzmann distribution. The concepts of $1 \mathrm{D}$ and 2D MRS are also presented in this chapter. The energy levels of two spin spin system, and expressions for the uncoupled and coupled 
case have been derived.

Chapter 3 introduces the various components of a clinical MR system. Various RF pulses, Coils and gradients have also been discussed. The need for volume localization, and current state-of-the-art clinical 1D and 2D MRS sequences have been discussed in detail.

Chapter 4 introduces multiple quantum coherences through a two spin $+\frac{1}{2}$ system. We also show that there are fewer multiple quantum transitions than single quantum transitions. The chapter starts with the product operator formalism which serves as the mathematical basis for the treatment of multiple quantum coherences. The various techniques for creating MQC's, properties of MQC's and coherence pathway selection are discussed.

Chapter 5 outlines the development of localized DQF-COSY for the GE 3T MRI / MRS Scanner in the EPIC environment. The creation and detection of double quantum coherences is described using the product operator formalism. The double quantum to single quantum pathway selection is achieved by issuing coherence selection gradients in the ratio 4:3:10.

Chapter 6 discusses the various data processing techniques used to improve the spectral resolution. The experimental results on a phantom containing ethanol, and another containing ethyl acetate are presented. To compare the performance the 1D, L-COSY spectrum has been presented with the localized DQF-COSY results. It is shown that the double quantum filter, effectively filters out all the single quantum coherences, and the resulting spectrum is much simpler than that of L-COSY. In vivo results on human calf muscle for the identification of EMCL and IMCL cross peaks have been presented.A Conclusion and future work is presented in Chapter 7 


\section{Chapter 2}

\section{One and Two Dimensional MRS}

\subsection{Magnetic Properties of the Nuclei}

The classical description of NMR provides physical insight into the behavior of the bulk magnetization, in terms of vector, manipulated during an NMR experiment. This approach is sufficient for explaining the physics of Magnetic Resonance that involves single quantum coherences (the spin quantum number changes by \pm 1 ). It is important to use the quantum mechanical description for explaining the physics of multiple quantum coherences (where the changes of the spin quantum number is greater than \pm 1$)$.

\subsubsection{Interaction of Magnetic Moment with Magnetic Field}

Atoms are made up of electrons and nuclei. An atomic nucleus is characterized by a number of parameters including atomic number, mass number and spin quantum number. The atomic number specifies the number of protons in the nucleus. The mass number specifies the total number of neutrons and protons in the nucleus. Nuclei that possess an odd number of protons and odd number of neutrons, or both, exhibit spin phenomena and have a nonzero spin angular momentum $I \bar{h}$, where $\bar{h}$ is given by $\bar{h}=\frac{h}{2 \pi}, I$ is the spin quantum number and $h$ is the Plank's constant. In accordance with the principles of quantum mechanics, spin angular momentum can take on the values $\frac{1}{2}, 1, \frac{3}{2}, 2, \frac{5}{2}$. In an external magnetic field, nuclei with a spin quantum number 
$I$ can assume one of $2 I+1$ possible eigen states (spin states, energy levels) related to the magnetic spin quantum number $m$, where $m$ can take values between $-I$ to I. The spin orientation in the presence of an external magnetic field is shown in Fig. 2.1 .

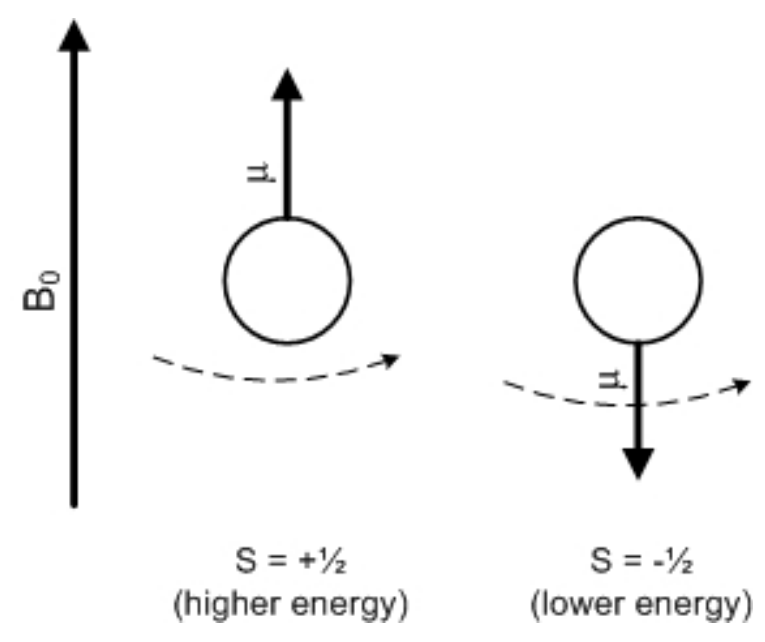

Figure 2.1: Spin Orientations

The magnetic dipole moment of a nuclear spin is given by

$$
\mu=\gamma \bar{h} I
$$

where $\gamma$ is the gyromagnetic ratio, which is characteristic of the nuclei. In an external magnetic field $\mathbf{B}_{0}$ the magnetic moment can take up to $2 I+1$ allowed orientations, each with its characteristic energy corresponding to the Zeeman Hamiltonian.

$$
\begin{aligned}
& H=-\mu B_{0} \\
& H=-\gamma \bar{h} I B_{0}
\end{aligned}
$$

\subsubsection{Precession}

The external magnetic field $B_{0}$ applies a torque on the magnetic moment $\mu$ shown in Fig. 2.2, which is inclined at angle $\theta$ to $B_{0}$. This causes the magnetic moment to rotate about the magnetic field with a frequency $\omega$ given by the Larmor Equation,

$$
\omega=\gamma B_{0} .
$$


The precession frequency is independent of the spin orientation, so all the spin orientations precess at the same frequency in a fixed magnetic field.

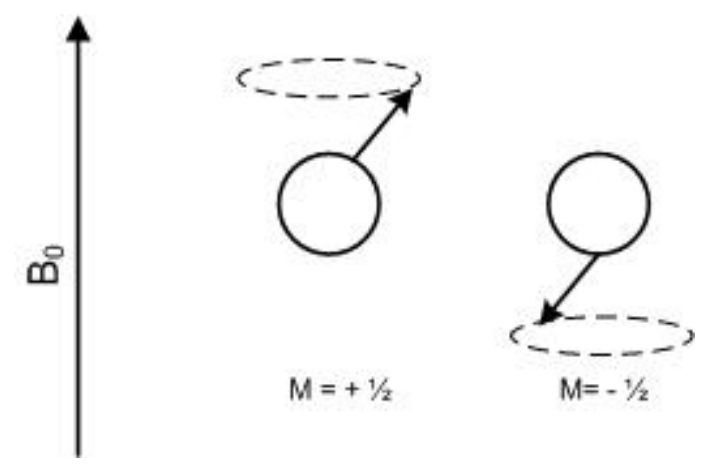

Figure 2.2: Precession Magnetic Moment

\subsubsection{Boltzmann Distribution}

According to principle of quantum mechanics, for net absorption to occur, the number of particles in the higher energy state should be greater than number of particles in the lower energy levels. If the two populations are equal, the probability of transition from higher to lower energy level is the same as that of transition from lower to higher energy level. For any system in thermal equilibrium, the number of particles in the lower energy level is higher than number in the higher energy levels. The Boltzmann equation gives distribution of particles at thermal equilibrium

$$
\frac{P_{m=-\frac{1}{2}}}{P_{m=+\frac{1}{2}}}=e^{-\frac{\Delta E}{k T}}=\exp \left(\frac{\Delta E}{k T}\right) .
$$

where $P$ is the population(fraction of particles) in each state, $T$ is the absolute temperature in Kelvin adn $k$ is the Boltzmann constant.

\subsubsection{The Rotating Frame of Reference}

When a system is placed in an external magnetic field $B_{0}$, the net magnetization $M$ aligns itself parallel to $B_{0}$. The net nuclear magnetization $M$ is the vector sum of individual magnetic moments. When an external magnetic field $B_{1}$ is introduced perpendicular to $B_{0}$ all the magnetization vectors become phase coherent and the 


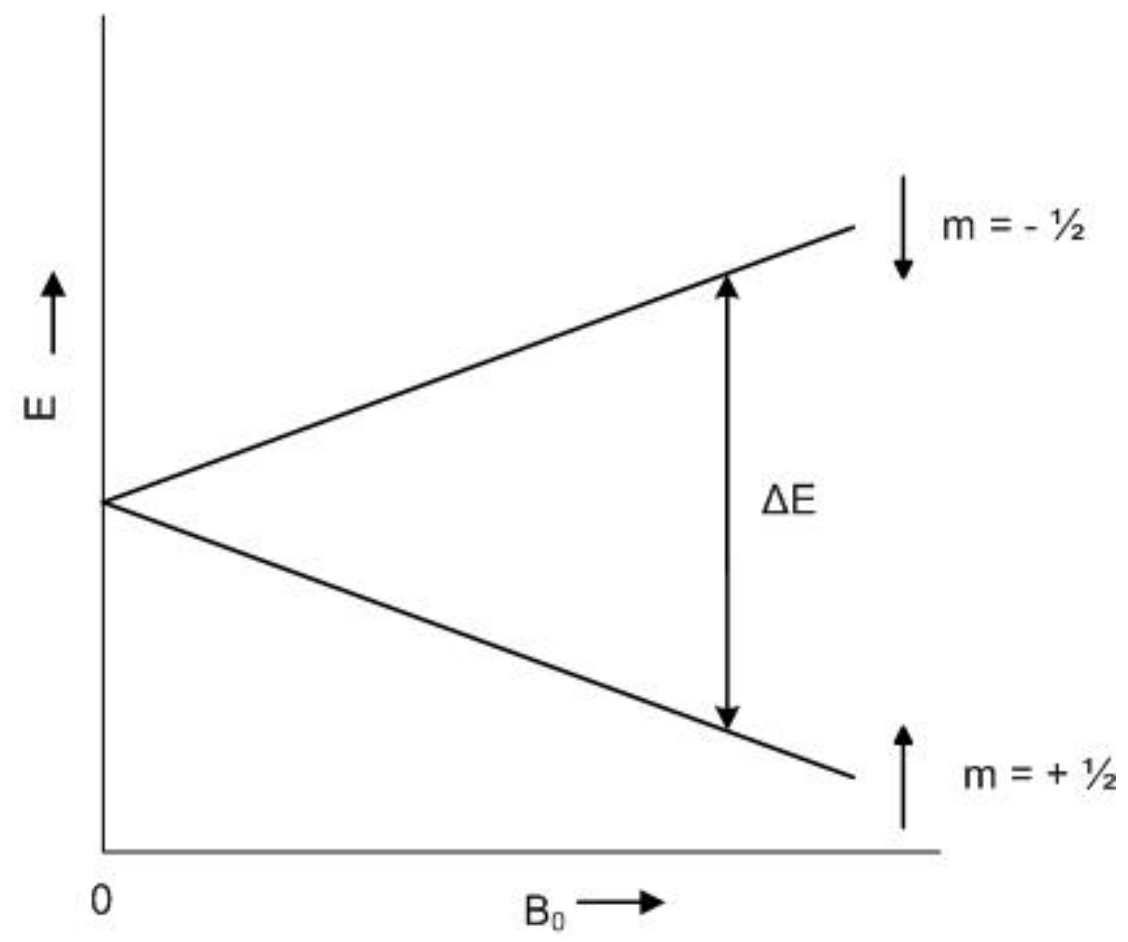

Figure 2.3: energy levels

magnetization $M$ precesses at angle $\theta$ to $B_{0}$. The magnetization $M$ precessing about the $z$ axis describing a cone. When the flip angle $\theta$ is $90^{\circ}$, the magnetization describes a disc on the $\mathrm{x}, \mathrm{y}$ plane. If we assume that the $x$ and $y$ axes were themselves precessing clockwise about the $z$ axis at the same frequency as the nuclear spins, the magnetization vector $M$ will appear stationary. To differentiate between the laboratory frame and the rotating frame, the axes are represented by $x^{\prime}, y^{\prime}$ and $z^{\prime}$ respectively. This rotating frame of reference will simplify the drawings of spins.

\subsection{One Dimensional MRS}

The signal in a one-dimensional experiment is recorded as a function of a single time variable, which is Fourier transformed to give a spectrum that is a function of a single frequency variable. The 1D spectrum contains chemical shift information (arising from chemically different ${ }^{1} H$ nuclei present in each metabolite) and spin-spin couplings (interaction due to different nuclear spins mediated by electron-nuclear and 


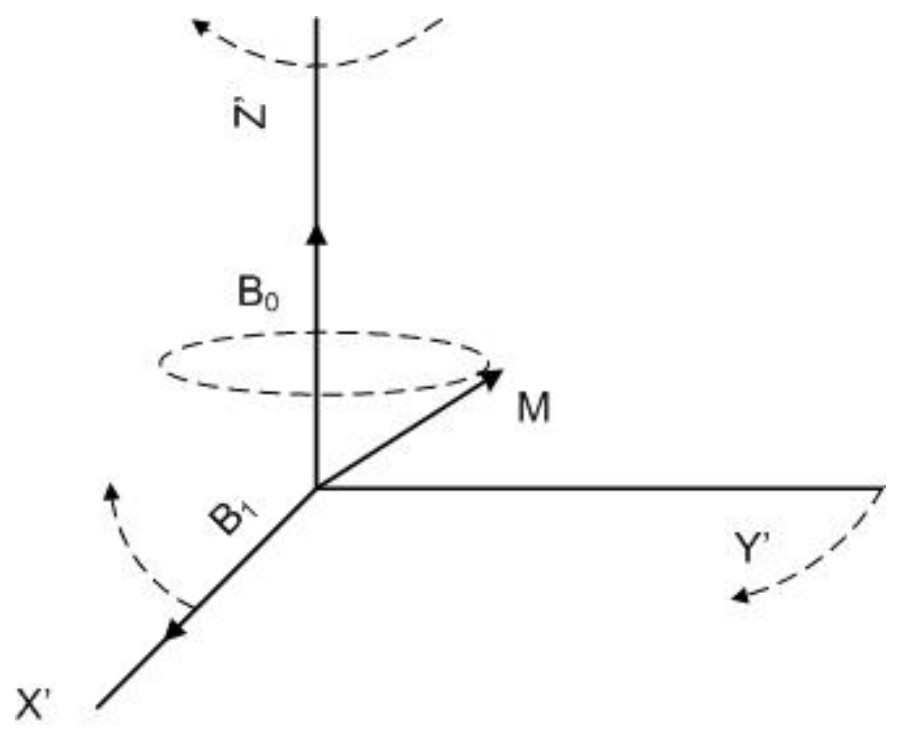

Figure 2.4: rotating frame of reference

electron-electron interactions) discussed below.

\subsubsection{Chemical Shifts}

The resonance frequency of an individual electron is influenced by the distribution of electrons in the chemical bonds of the molecule. The different chemical environment of the protons in the molecule leads to NMR signals appearing at different resonant frequencies. This is known as the chemical shift.

The applied magnetic field $B_{0}$, induces circulations in the electron cloud surrounding the nucleus such that, following Lenz' law, a magnetic moment $\mu$, opposed to $B_{0}$ is produced. Thus the field experienced by the proton may be lower than the applied field. This is equivalent to magnetic shielding of the nucleus that reduces $B_{0}$ by an amount equal to $\sigma B_{0}$ where $\sigma$ is known as the shielding constant of the particular proton

$$
B_{\text {local }}=B_{0}(1-\sigma)
$$

The shielding effect is shown in Fig 2.5. It is very difficult to specify the reso- 


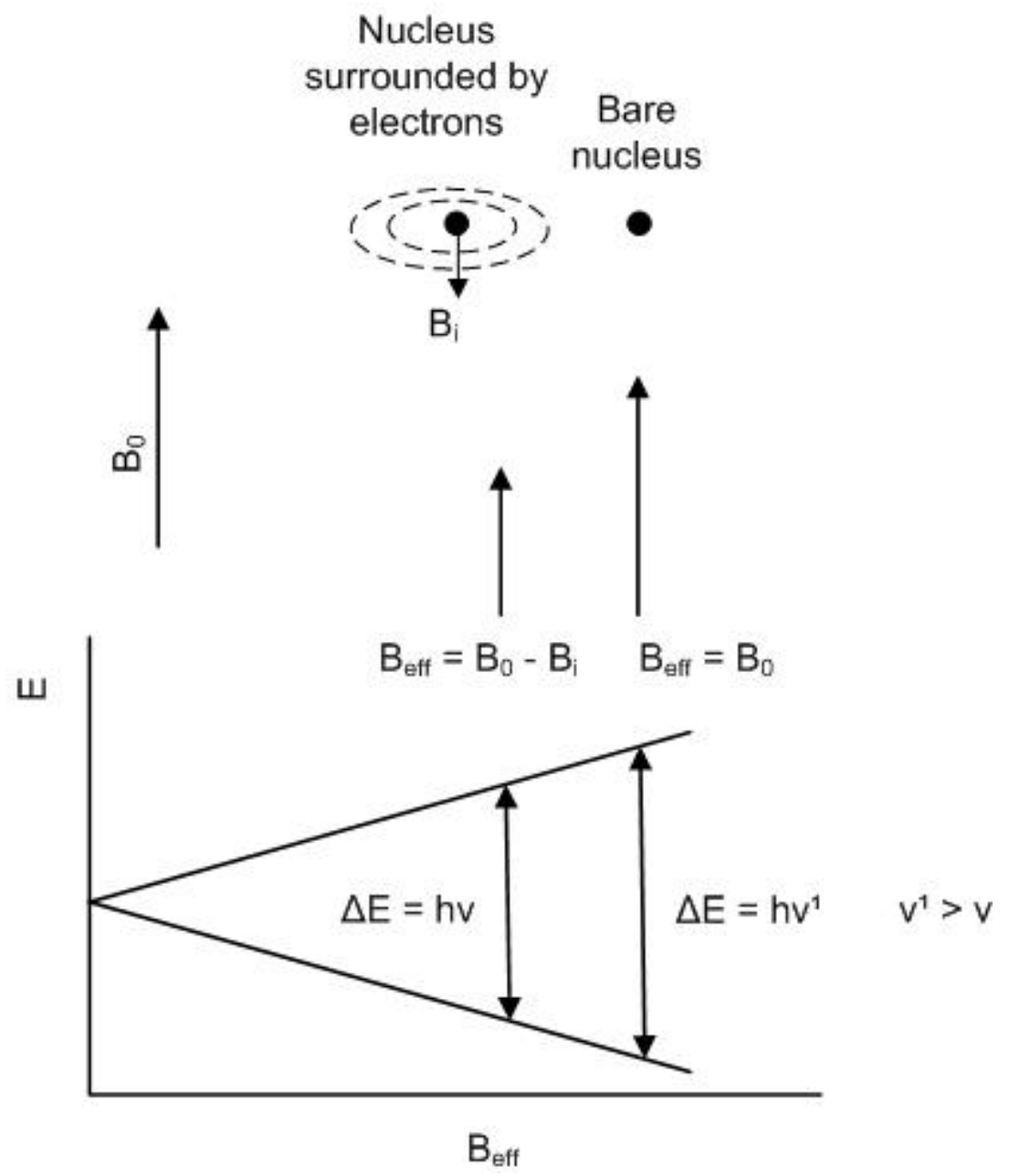

Figure 2.5: Shielding Effect

nance frequency. This is due to the fact that accurately measuring the external field strength, and the resonance frequency is not possible. Hence resonance frequencies are measured relative to that of a reference compound. A dimensionless quantity has been introduced to characterize the chemical shift. This is defined as

$$
\delta=\frac{\left(\nu_{\text {substance }}-\nu_{\text {reference }}\right)}{\nu_{0}}
$$

where $\nu_{0}$ is the operating frequency of the spectrometer. Thus $\delta$ is reported in ppm (parts per million) of the operating frequency. 


\subsubsection{Spin-Spin Coupling}

The individual signals expected based on the molecule's symmetry are found to split into symmetrical patterns (multiplets) consisting of two or more lines. This interaction between individual nuclear spins is caused by the magnetic interaction through chemical bonds mediated by the electrons. These multiplets offer valuable structural information about the molecule. The multiplicity of a signal is the number of lines in the signal and can be determined by $2 n I+1$, where is $n$ is the number of protons. The energy, $E$, of the spin-spin interaction between two nuclei is proportional to the scalar product of their nuclear magnetic moments. The energy of the coupling and consequently the coupling constant, unlike the chemical shift, is independent of the strength of the magnetic field. These constants are therefore expressed in frequency units $(\mathrm{Hz})$. Fig. 2.6 shows chemical shift and spin-spin coupling information.

\subsection{Energy Level of a Two Spin System}

\subsubsection{Energy Levels}

It is convenient to label the energy levels according to magnetic quantum number, $m_{I}$ which can take values from $+I$ to $-I$ in integer steps. Each $m_{I}$ value corresponds to a different orientation of the magnetic moment with respect to the magnetic field. the energy of each level is given by

$$
E\left(m_{I}\right)=\frac{-m_{I} \gamma B_{0} h}{2 \pi}
$$

where $\mathbf{B}_{0}$ is the external magnetic field, $h$ is Plank's constant and $\gamma$ is the gyromagnetic ratio which is is constant for a nuclear species. The magnetic dipole transitions have the energy

$$
\Delta E=\frac{\gamma B_{0} h}{2 \pi}
$$

which can be simplified into

$$
\nu_{L}=\frac{\gamma B_{0}}{2 \pi}
$$




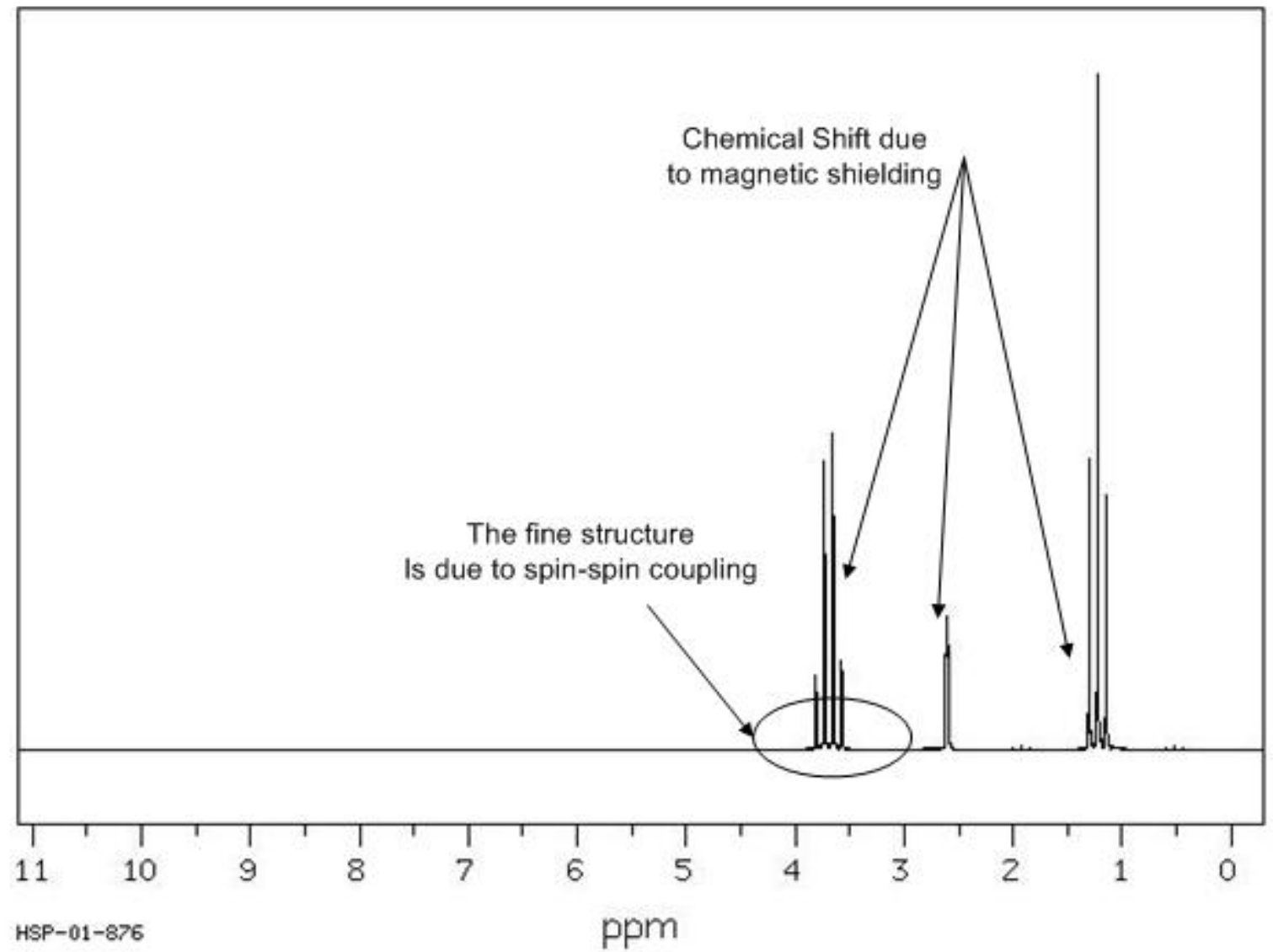

Figure 2.6: 1D Spectrum of Ethanol showing chemical shifts and spin-spin coupling

where $\nu_{L}$ is the Larmor precession frequency. When the chemical shift is considered, this expression gets slightly modified

$$
\nu_{L}=\frac{\gamma B_{0}(1-\sigma)}{2 \pi}
$$

Where $\sigma$ is the nuclear shielding parameter. In a single spin $\frac{1}{2}$ system, the spins can be aligned parallel $\left(m_{I}=+\frac{1}{2}\right)$ or antiparallel $\left(m_{I}=-\frac{1}{2}\right)$ to the external magnetic field $\mathbf{B}_{0}$. The two spin states are labelled $\alpha$ and $\beta$ respectively. When a photon of energy equal to that of $\Delta E$ is applied, it causes the spin to transition from the lower energy level $\beta$ to higher energy level $\alpha$. The external energy applied is absorbed in the form of a spin flip. 


\subsubsection{Two Spin System}

Let us consider a spin system with two coupled spins $I$ and $S$, where $S$ is the spin to be observed. The nuclear spins interact with one another by spin-spin coupling, which acts through the electrons in the chemical bonds. The nuclear spin $S$ is influenced by the presence of a neighboring spin $I$. The spin $I$ generates a small magnetic field, which may add to or subtract from the main magnetic field $\mathbf{B}_{0}$ depending on whether the spin is aligned parallel $(\alpha)$ or antiparallel $(\beta)$ to the external magnetic field $\mathbf{B}_{0}$. This splits the $S$ resonance frequency peak into a doublet with splitting $J_{I S}(H z)$ where $J_{I S}$ is the coupling constant. The $I$ spin experiences a similar splitting, due to the presence of neighboring spin $S$. Unlike the chemical shift, spin-spin coupling is independent of the external magnetic field $\mathbf{B}_{0}$ because it is a built in physical interaction within the molecule, whereas the chemical shift arises due to the nuclear shielding effect which reduces the magnetic field experienced by the nucleus.

Let us consider two spins $I$ and $S$ with Larmor frequencies $\nu_{I}$ and $\nu_{S}$ with zero spin-spin coupling.

$$
\begin{gathered}
\nu_{I}=\gamma B_{0}\left(1-\sigma_{I}\right) / 2 \pi \\
\nu_{S}=\gamma B_{0}\left(1-\sigma_{S}\right) / 2 \pi
\end{gathered}
$$

we can safely assume $\nu_{I}>\nu_{S}$ without loss of generality. There are four possible energy levels.

$$
\begin{array}{ll}
\text { IS } & \text { Energy } \\
\hline \beta \beta & E_{4}=+\frac{1}{2} \nu_{I}+\frac{1}{2} \nu_{S} \\
\beta \alpha & E_{3}=+\frac{1}{2} \nu_{I}-\frac{1}{2} \nu_{S} \\
\alpha \beta & E_{2}=-\frac{1}{2} \nu_{I}+\frac{1}{2} \nu_{S} \\
\alpha \alpha & E_{1}=-\frac{1}{2} \nu_{I}-\frac{1}{2} \nu_{S}
\end{array}
$$

if we introduce a non-zero spin-spin coupling $J_{I S}$ the energy levels change slightly. 


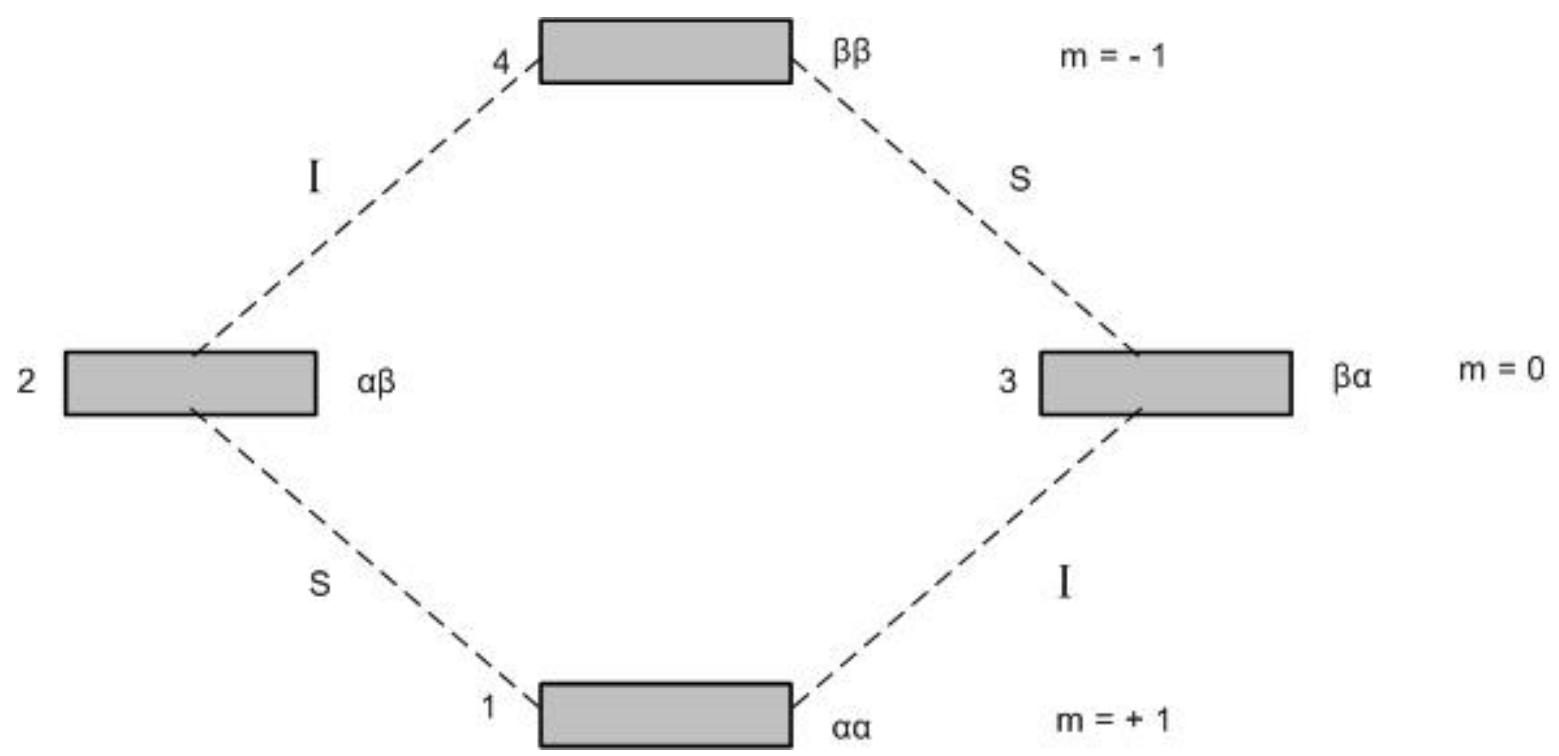

Figure 2.7: Energy level diagram of a coupled two spin system

\begin{tabular}{ll} 
IS & Energy \\
\hline$\beta \beta$ & $E_{4}=+\frac{1}{2} \nu_{I}+\frac{1}{2} \nu_{S}+J_{I S}$ \\
$\beta \alpha$ & $E_{3}=+\frac{1}{2} \nu_{I}-\frac{1}{2} \nu_{S}-J_{I S}$ \\
$\alpha \beta$ & $E_{2}=-\frac{1}{2} \nu_{I}+\frac{1}{2} \nu_{S}-J_{I S}$ \\
$\alpha \alpha$ & $E_{1}=-\frac{1}{2} \nu_{I}-\frac{1}{2} \nu_{S}+J_{I S}$
\end{tabular}

The sign of $J_{I S}$ depends on spin states of both the nuclei. The sign of $\nu$ depends on the spin state of one nucleus. If we assume $\left(\nu_{I}-\nu_{S}\right) \gg\left|J_{I S}\right|$ this is known as weak coupling. $m$ is the total magnetic quantum number. Since $\nu_{I}$ and $\nu_{S}$ are very much larger than $\left|\nu_{I}-\nu_{S}\right|$ and $\left|J_{I S}\right|$, the separations of levels 2 and 3 are negligible.

The single quantum transitions have $\delta m= \pm 1$, and are (1) - (3), (2) - (4), (1) - (2) and $(3)-(4)$. The first two transitions involve a change in the $I$ spin quantum number and are thus $I$ spin transitions.

$$
\begin{aligned}
& \nu_{13}=\nu_{I}-\frac{1}{2} J_{I S} \\
& \nu_{24}=\nu_{I}+\frac{1}{2} J_{I S}
\end{aligned}
$$

second two are S-spin transitions.

$$
\begin{aligned}
& \nu_{12}=\nu_{S}-\frac{1}{2} J_{I S} \\
& \nu_{34}=\nu_{S}+\frac{1}{2} J_{I S}
\end{aligned}
$$


without loss of generality we can assume $J_{I S}>0$. The results can be generalized to the case of $\mathrm{N}$ spin $+\frac{1}{2}$ nuclei.

$$
E=-\sum_{i=1}^{N} m_{i} \nu_{i}+\sum_{i>j}^{N} m_{i} m_{j} J_{i j}
$$

where the second summation is over all of unlike spins $i$ and $j, \nu_{i}$ is the Larmor frequency of the $i t h$ spin and $J_{i j}$ is the spin-spin coupling between spins $i$ and $j$.

\subsubsection{Energy Level Populations}

For a simple two level system at equilibrium, the energy levels are populated according to the Boltzmann equation.

$$
N_{\text {upper }} / N_{\text {lower }}=\exp (-\Delta E / k T)
$$

For NMR transitions practical field strengths and temperatures, $\Delta E \ll k T$ and the exponential can be expanded to first order in $\Delta E$, giving

$$
\begin{aligned}
& N_{\text {upper }} / N_{\text {lower }}=1-\Delta E / k T \\
& \frac{\left(N_{\text {lower }}-N_{\text {upper }}\right)}{N_{\text {lower }}}=\Delta E / k T
\end{aligned}
$$

The above equation implies that population differences are directly proportional to energy gaps. With this approximation, the equilibrium population differences for all proton (single-quantum) transitions may be put equal, since we can safely disregard the very small changes in $\Delta E$ due to chemical shifts.

Since the probabilities of stimulated emission and absorption are equal, the absorption of energy due to upward transitions is largely cancelled by the emission of energy due to the downward transitions. Hence, the observed signal depends on only the population difference $\Delta$. The populations may be perturbed by RF pulses, and a population inversion pulse (often called a $180^{\circ}$ or a $\pi$ pulse), moves all the spins from the lower energy level to higher energy level. 


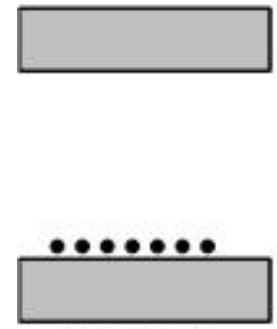

Boltzmann
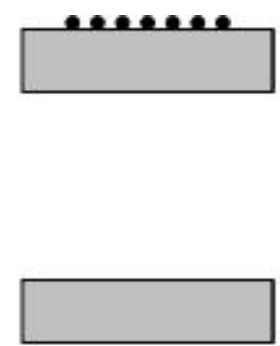

Inversion

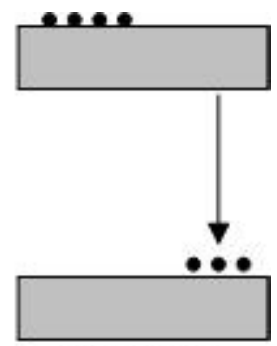

Relaxation

Figure 2.8: Boltzmann distribution of spins

\subsection{Introduction to 2D MRS}

The interpretation of in vivo NMR spectra is often impeded by the complexity of overlapping resonances. This is especially true for in vivo ${ }^{1} H$ NMR spectra, in which many (scalar-coupled) resonances overlap in a small chemical shift range of less than $10 \mathrm{ppm}$. The difference in characteristics of resonances such as chemical shift, couplings, parameters like relaxation, diffusion etc can be used to selectively observe the desired resonance signals by suppressing the undesired signals. The undesired signals can be suppressed by various 1D techniques, which use one of the above-mentioned properties. However all these methods lead to some loss of signal. A better way of interpreting the NMR data is to separate the various characteristics along orthogonal axes by multidimensional NMR.

The first two-dimensional NMR experiment, (homonuclear) correlation spectroscopy (COSY) was designed by J. Jeener $[27\rfloor$ to obtain information about homo nuclear coupling connectivities for identification of scalar coupled spin systems.

The general idea of $2 \mathrm{D}$ experiments is to generate a second frequency axis by introducing an evolution delay into a pulse sequence, during which the transverse magnetization precesses at a different frequency from that during signal acquisition.

For each value of $t_{1}$, the same pulse sequence is executed and a free induction decay (FID) consisting of $\mathrm{n}$ data points is recorded during the $\mathrm{t} 2$ period. Thus the 
signal is acquired as a function of two separate independent time periods $\left(t_{1}\right.$ and $\left.t_{2}\right)$. The rows of the data matrix represent data collected for a fixed $t_{1}$ value and a different $t_{2}$ value; the columns represent data collected for a fixed $t_{2}$ value and varying $t_{1}$ values. Fourier transformation of the data with respect to these two time domains generates a $2 \mathrm{D}$ spectrum using two independent frequency dimensions $F_{1}$ (from $t_{1}$ ) and $F_{2}$ (from $t_{2}$ ). The signal recorded during $t_{2}$ is modulated by events occurring during the evolution time $t_{1}$. 


\section{Chapter 3}

\section{Clinical MRS}

\subsection{Introduction to Clinical MR System}

The MRI System is complex system consisting of various components, which act in relation with each other. The various components are

- Magnet System

- Gradient System

- RF System

- Patient Table

- Control System

- Main Computer

- Supporting Systems

The MR magnet is located inside the scanning room, which is RF shielded. The main magnet produces the static magnetic field, $B_{0}$. The gradient coils are used to produce gradient magnetic fields in the three dimensions. The RF coil is used to produce the external magnetic field $B_{1}$, which is used to excite the protons in the subject. The gradient coils and the RF coils are placed inside the magnet. The RF coil can act as a receiver and it picks up the signal generated by the subject. The 
RF shielding prevents the signal from external RF sources such as radio and television from reaching the scanner. It also prevents the RF signals generated inside the scanner room from leaking outside. There are safety issues due to the interference of magnetic field with pacemakers, inplants etc. Therefore magnets are also shielded to prevent the spread of magnetic field outside the required area.

\subsubsection{Magnet System}

There are generally three types of magnets used in MR research.

Permanent Magnets This type of magnet is always magnetized. The advantages of these magnets are that, they don't require any external energy to keep their magnetization. The disadvantage is that these magnets are highly sensitive to thermal instability and require temperature control. They also produce limited field strengths.

Resisitive Magnets These are electromagnets and require electricity flowing through them to keep them magnetized. As the coils are resistive, they generate heat and require a cooling arrangement. Generating high magnetic field strengths are not possible, as they require high electric energy and elaborate cooling arrangements.

Superconductive Magnets These are the type of magnets used in most of the MR systems. Superconductivity is achieved by immersing the coil made up of Niobium-Titanium wire in liquid helium. The liquid helium keeps the coil temperature at $4.2 \mathrm{~K}$. At this temperature the resistance of the coil is effectively zero, and no heat loss occurs when electric current is flowing through it. Ideally, the electric current flowing through the coil will be flowing forever, creating a constant magnetic field. If the temperature of the coil rises by a few degrees, there will be a loss of superconductivity. In this case there will be rapid heat production due to resistance to current flow, resulting in the sudden boil off of 
liquid helium. This will render the magnet discharged and most of the helium will escape to the atmosphere. High magnetic fields can be produced using superconductive magnets. Most of the commonly used magnets are $1.5 \mathrm{~T} / 3.0 \mathrm{~T}$ for clinical applications. These magnets produce highly homogenous magnetic fields. The scanner used in this research uses a 3T magnet.

\subsubsection{Magnetic and RF Shielding}

To prevent the spread of magnetic fields, outside the desired area, the magnetic field is shielded. The scanner room may be covered by ferromagnetic pieces, which will concentrate the magnetic field inside the scanner room. This is called passive shielding. Newer magnets uses another superconducting coil located inside, which produces a magnetic field opposite to the polarity of the main magnetic field in the area outside the imaging area. This is called active shielding. The scanner operates in the RF frequency range. To prevent the scanner from interfering with external $\mathrm{RF}$ devices, and to prevent external RF devices interfering with the working of the scanner, it is shielded.

\subsubsection{Shimming}

The fields produced by all types of magnets are not completely homogenous. The process of making this field homogeneous is called shimming. This can be achieved by placing small iron or magnetic pieces in the magnetic field. This is called passive shimming. The other option is to place small current carrying coils inside the magnet, whose currents may be adjusted to make the fields homogenous. This is called active shimming.

\subsubsection{Gradient System}

The gradient coils produce linearly varying magnetic fields, in three dimensions. Slice selection and spatial information is possible because of gradient coils. The strength, duration and timing of the current, depends on the type of study to be 
performed. These values are determined by the system operator. Sudden and frequent changes of magnetic field due to gradients cause eddy currents to be produced in various metallic parts of the magnet. Actively shielded gradients coils minimize the production of these eddy currents.

\subsubsection{RF System}

MRI systems require a transmitter and receiver. A transmitter is used to produce RF to excite the protons. The transmitter is made up of an RF generator, that produces a small RF signal. This signal is then shaped in the desired form, using modulator and waveform generator, to generally the shape of a sinc pulse. This signal is amplified using an RF amplifier, and then radiated to the subject by the RF coil. After excitation of the protons in the subject, the RF pulse transmission is stopped. The protons in the subject return back to their original state releasing RF energy. This energy is captured by the receiver coils, which are kept closer to the subject. The transmission of RF is done by the body coil, which is permanently fixed in the scanner. The receiver coil is connected as required depending on the area to be examined. The receiver section generally has a preamplifier, amplifier, demodulator and an $\mathrm{A} / \mathrm{D}$ converter.

\subsubsection{Patient Table}

The patient table is computer controlled and is used to move the patient into the imaging area. The movement of the table can be controlled from the operator console. Connections for various coils are incorporated into the table.

\subsection{RF Pulses}

Radio frequency $(\mathrm{RF})$ pulses are used in various spin manipulations like excitation, inversion and refocusing. The RF pulse must have the same frequency as that of the precession frequency (Larmor frequency) of the nuclei under investigation. RF 
pulses can generally be classified into two types, hard and soft pulses. Short, intense, constant-amplitude pulses (hard pulses) are used to achieve uniform excitation of the spin system. Most of the in vivo experiments require more sophisticated RF pulses. Instead of exciting the entire spin system, a well-defined limited frequency range has to be excited. The design of these RF pulses can be done using Fourier transform theory, Bloch equations or optimization procedures. When the amplitude of the RF pulse is strong enough such that off-resonance effects $\Delta \Omega$ (the difference between the Larmor frequency $\omega_{0}$ and the frequency $\omega$ of irradiating field $B_{1}$ ) can be neglected, i.e. $\left|\gamma B_{1}\right| \ggg|\Delta \Omega|$, the nutation angle $\theta$ is given by

$$
\theta=\gamma B_{1} T
$$

where $\mathrm{T}$ is the duration of the pulse. The Shinnar-Le Roux (SLR) algorithm $[28$, 29, 30, 31, 32, 33 for designing RF pulses is used in the GE Horizon LX Scanners. The SLR algorithm is used to design the RF pulse profile from the desired frequency response. It maps the RF pulse design problem into a polynomial design problem. The optimization permits the generation of pulses with slice profiles having no side lobes and very small out-of-slice ripples.

\subsection{RF Coils}

The RF coils in MRI/ MRS are generally used for two purposes. The coils which excite the magnetization are called transmit coils. The coils which receive signal from the excited spins are called receive coils. The same coil can be used to transmit and receive the signal, but often separate coils are used for excitation and detection. The GE Signa Horizon LX 3T scanner comes with a built in transmit coil which can excite the whole body. Desirable characteristics of a RF transmit coil include homogeneity of magnetic field and minimization of time needed to tip the spins away from the static magnetic field.

A Birdcage coil is the most popular coil configuration in MRI / MRS. These coils 
have a quadrature design and excellent radial field homogeneity over the ROI (Region of Interest). If the ROI is localized near the surface of the body, a surface coil may be used. The SNR is maximized because the coil is placed very close to the object being imaged. A set of coils, whose signals are combined to obtain a uniform signal is called a phased array coil. Phased array coils take advantage of the high SNR available from smaller coils.

\subsection{Volume Localized MRS}

\subsubsection{Need for Localization}

Samples in biological tissues are spatially inhomogeneous in terms of metabolic composition and magnetic field strengths. For adequate tissue characterization it is necessary to employ a spatial localization technique such that the origin of the NMR signal is accurately known without contamination from other spatial positions. For localized detection of metabolites data acquisition schemes are combined with water suppression techniques. There are a variety of localization methods. The most important among them are $B_{1}$ and $B_{0}$ gradient based localization techniques. The $B_{1}$ dependence of the nutation angle of square RF pulses is used to obtain spatial information. $B_{0}$ based gradient methods rely on the selection of a spatially selective slice by the application of a frequency - selective RF pulse in the presence of a $B_{0}$ magnetic field gradient. The techniques can be broadly classified into outer volume suppression, where the magnetization in the volume-of-interest is left unperturbed during localization and those which selectively excite the magnetization in order to remove unwanted magnetization outside the volume-of-interest.

\subsubsection{Water Suppression}

The human body contains $60 \%$ water. The concentration of water in human tissue is in the order of $110 \mathrm{M}$, while the concentration of the metabolites of interest are in the range of 0.1 to $0.2 \mathrm{mM}$. It is impossible to observe the weak metabolites in the 
presence of water, due to the high concentration of water. Hence the resonance of water needs to be suppressed to observe the metabolites.

The suppression of a particular resonance in the NMR spectrum requires a difference in the property between the protons in the molecule under investigation, and the compound interfering with the detection. The nuclear properties such as chemical shifts, scalar couplings and relaxation can be used to achieve suppression. Other non-nuclear properties such as diffusion and exchange can be used, but they should reflect in the NMR Spectrum as an observable parameter. Fig. 3.1 shows the water suppression waveform used in GE 3.0T Signa Horizon LX Scanner.

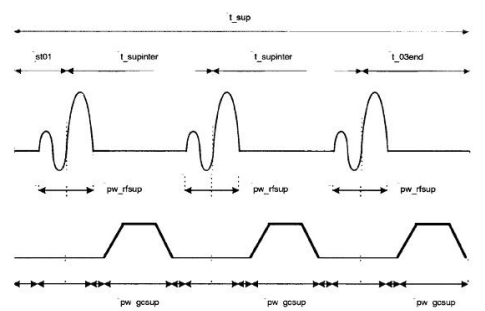

Figure 3.1: Water suppression in GE 3.0T Signa Horizon LX Scanner. Reproduced from $[1]$.

\subsubsection{Outer Volume Suppression}

Outer volume suppression pulses remove unwanted spins during a preparation period prior to the excitation of the desired spins. The transverse magnetization that is parallel to the coil is dephased by the special gradient. This process is generally repeated several times to improve suppression. Outer volume suppression is used in all the spectroscopic sequences by default. The outer volume pulses used in the GE 3.0T Horizon LX Scanner are shown in Fig. 3.2 The widths of the saturation bands are equal to the corresponding dimensions of the prescribed voxel(small volume of interest). The center of the saturation bands is placed at one-half of the width of the bands from the edges of the voxel. The chemical shift artifact along each axis is similar for both the voxel and the bands, thereby minimizing possible saturation of a 


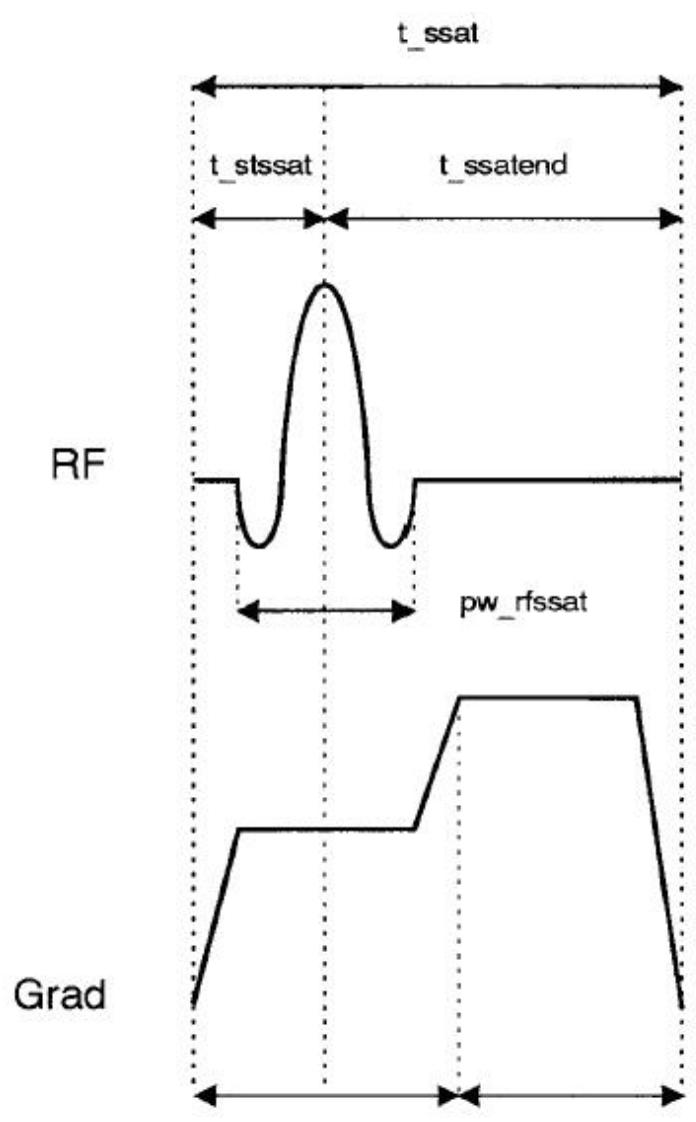

Figure 3.2: Outer volume suppression in GE 3.0T Signa Horizon LX Scanner. Reproduced from $[1]$.

portion of the spectrum from within the voxel by the saturation pulses.

\subsection{Single Voxel Localization}

The popular methods of single voxel localization are ISIS, STEAM and PRESS. Single voxel localization is achieved by using three RF pulses in the presence of slice selective gradients, which select planes along three orthogonal directions. The intersection of these planes produces a small voxel.

\subsubsection{Image Selected In Vivo Spectroscopy (ISIS)}

The ISIS sequence [34] employs selective inversion pulses in the presence of gradients, which are turned on and off according to an encoding scheme as show in Fig. 
3.3. When zero or an even number of pulses are executed, the desired magnetization

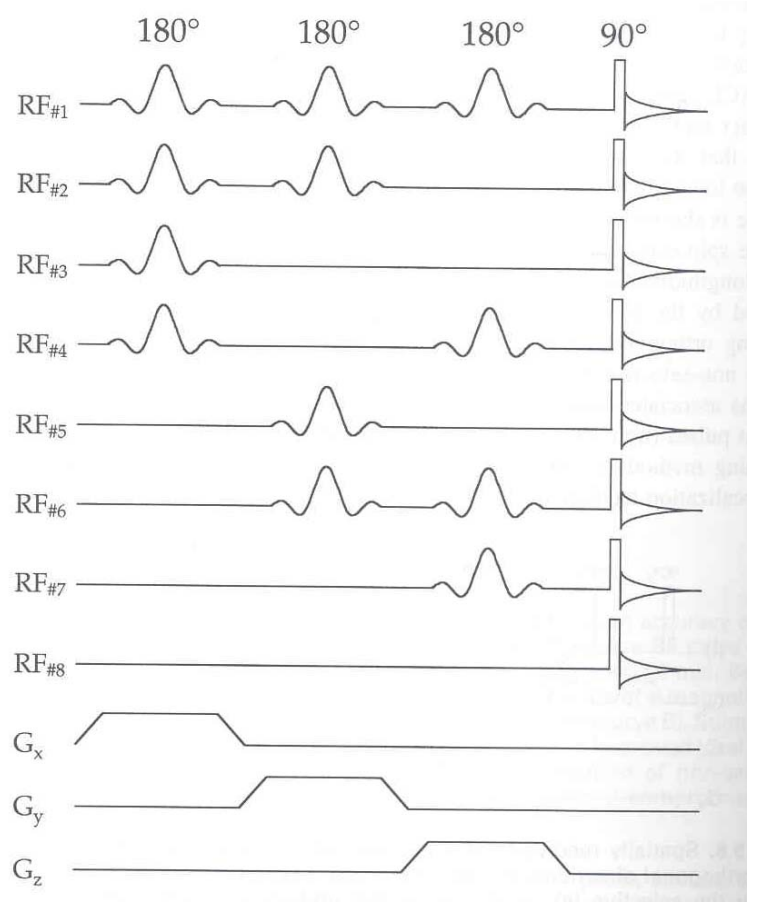

Figure 3.3: Image-selected in vivo spectroscopy (ISIS). Reproduced from $\lfloor 2\rfloor$

is rotated to the positive longitudinal axis prior to the excitation and will coherently add to the signal from the localized volume. When an odd number of inversion pulses are employed, the desired magnetization is inverted and needs to be subtracted during the data collection. The unwanted magnetization completely cancels out when the phase cycle is complete. Because the magnetization remains in the longitudinal axis during ISIS, this localization technique is relatively insensitive to T2 relaxation. The chemical shift induces frequency offsets, and inhomogeneity can degrade the performance of the inversion and excitation pulses.

\subsubsection{STimulated Echo Acquisition Mode (STEAM)}

STEAM (Stimulated Echo Acquisition Mode) is a localization technique [35] capable of complete three-dimensional localization in a single acquisition. i.e. STEAM is a single-shot or single-scan localization scheme. The pulse sequence is shown in Fig. 3.4. The basic pulse sequence (without the $B_{0}$ magnetic field gradients) consists of 
three RF pulses with a nominal nutation angle of $90^{\circ}$. This pulse sequence generates five echo's and three FIDs as described by Hahn [36]. When the first two RF pulses are separated by a delay of $\frac{T E}{2}$ and the last two pulses (pulses 2 and 3) are separated by a delay of TM, four spin-echoes are formed. The spin-echo formed by pulses 1 and 2 (SE12) occurs at the position TE after the initial excitation pulse, SE13 at position at TE $+2 \mathrm{TM}, \mathrm{SE} 23$ at position $\frac{T E}{2}$ and SE123 (due to refocusing by pulses 2 and 3 of excited magnetization by pulse 1) occurs at position $2 T M$. Besides these four spin echoes a stimulated echo is formed at position $T E+T M$. Fig. 3.4 shows the various echoes formed during a sequence containing three RF pulses.

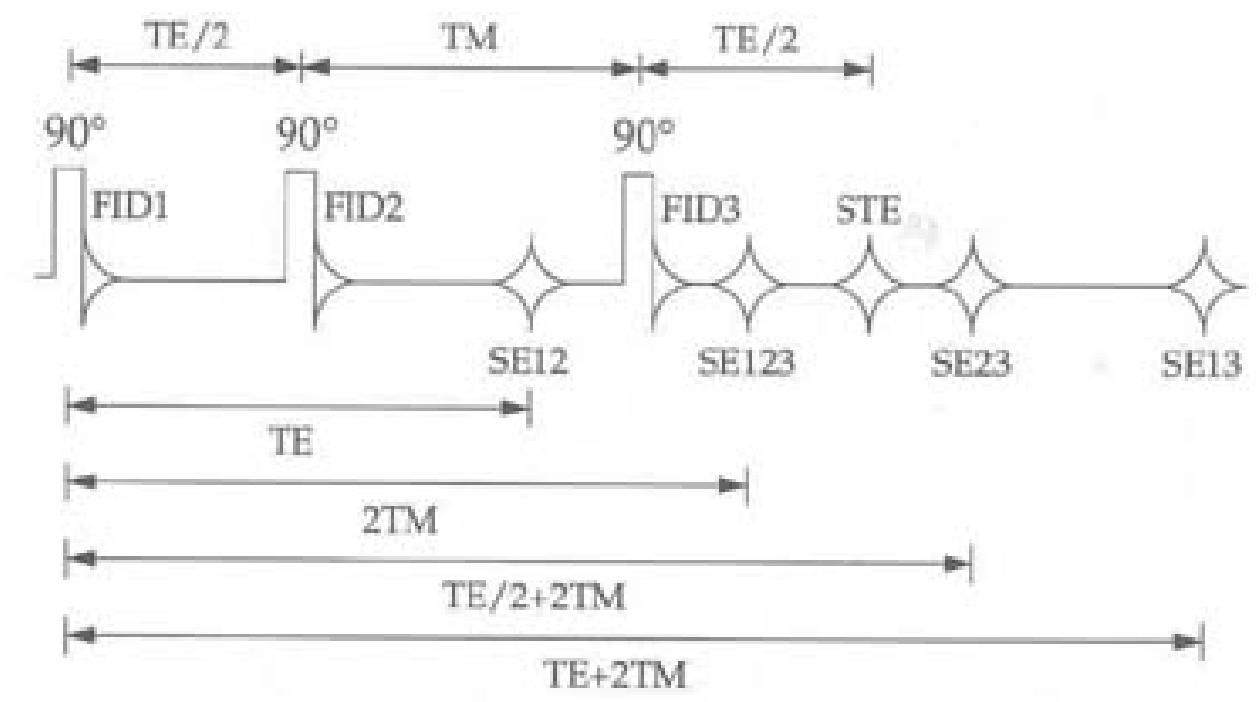

Figure 3.4: STEAM pulse sequence. Reproduced from $[2]$

In spectroscopy experiments the stimulated echo is the signal of interest. Therefore the FID and the spin echoes needs to be eliminated to obtain a clean signal from the stimulated echo. This can be achieved by phase cycling the individual RF pulses. This will degrade the single-scan character of STEAM localization. Also, phase cycling can achieve good results on stable systems, which is not always the case with in vivo spectroscopy. Alternatively, the unwanted FID and spin-echo signals can be eliminated with the use of $B_{0}$ magnetic field gradients. By placing a $B_{0}$ crusher gradient in the TM period, all spin-echoes are eliminated leaving only a FID compo- 
nent arising from the last excitation pulse. This FID can be eliminated by placing a $B_{0}$ crusher gradient in the first $\frac{T E}{2}$ delay and an identical $B_{0}$ gradient in the last $\frac{T E}{2}$ period. The amplitude of a stimulated echo is theoretically $50 \%$ of a spin echo. The $50 \%$ reduction originates from the fact that the second $90^{\circ}$ pulse only rotates half of the transverse magnetization to the longitudinal axis, while the other half is dephased by the TM crusher. Practically the amplitude might be a little lower than this due to different sensitivities of the localization techniques to relaxation, diffusion and J-coupling effects.

\subsubsection{Point RESolved Spectroscopy (PRESS)}

The PRESS (Point RESolved Spectroscopy) localization method $[37,38,39,40]$ is directly derived from DRESS [41]. In DRESS one-dimensional localization is achieved by a frequency-selective RF pulse in the presence of a $B_{0}$ gradient. In PRESS two frequency-selective $180^{\circ}$ pulses are added. When these additional RF pulses are executed during $B_{0}$ magnetic field gradients, the sequence is capable of three-dimensional localization. When the first $180^{\circ}$ pulse is executed at time $t_{1}$ after the excitation pulse, a spin echo is formed at time $2 t_{1}$. The second $180^{\circ}$ pulse refocuses this spin-echo during a delay $2 t_{2}$, such that the final spin-echo is formed at time $t_{1}+2 t_{2}$ (which equal the echo time of PRESS i.e $T E=2 t_{1}+2 t_{2}$ ) The first echo contains the signal from a column, which is the intersection of the two orthogonal slices selected by the $90^{\circ}$ pulse and first $180^{\circ}$ pulse. The second spin-echo contains signal only from the intersection of the three planes selected by the three pulses resulting in the desired volume. Signals outside the volume of interest is neither excited nor refocused leading to rapid dephasing of the signal. The PRESS sequence is shown in Fig. 3.5.

Because PRESS sequence employs $180^{\circ}$ refocusing pulses, there is no mechanism present for creating multiple quantum coherences in coupled spin systems as in the case of STEAM sequence. 


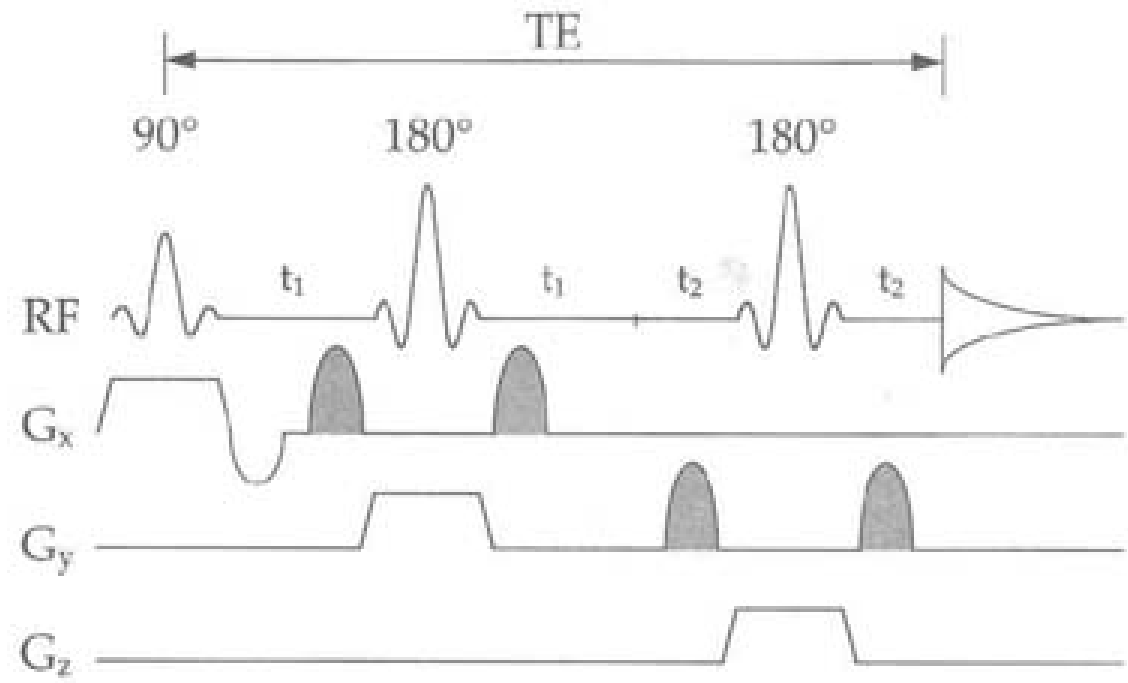

Figure 3.5: PRESS pulse sequence. Reproduced from $[2\rfloor$

\subsection{Limitations of $1 \mathrm{D}$ MRS}

The simulated 1D spectra of various metabolites are shown in Fig. 3.6. The spectra of resulting metabolites overlap heavily and this prevents complete characterization. Moving to higher field strengths will resolve the metabolites, but this is not possible in the clinical MR field. Hence there is a need for other techniques which will help to characterize these metabolites.

\subsection{Localized 2D MRS}

In the regular one-dimensional (1D) NMR spectrum all information is contained in two directions, frequency and intensity. The 1D spectrum does not provide any information about which spin is coupled to which other spin. For complex molecules it may not be possible to disentangle the spin-coupling network via 1D double resonance experiments. A 2D NMR spectrum is collected as a function of two time variables [42]. The resulting spectrum will have two frequency axes, obtained via two-dimensional Fourier transformation of time-domain data with two orthogonal time axes. 


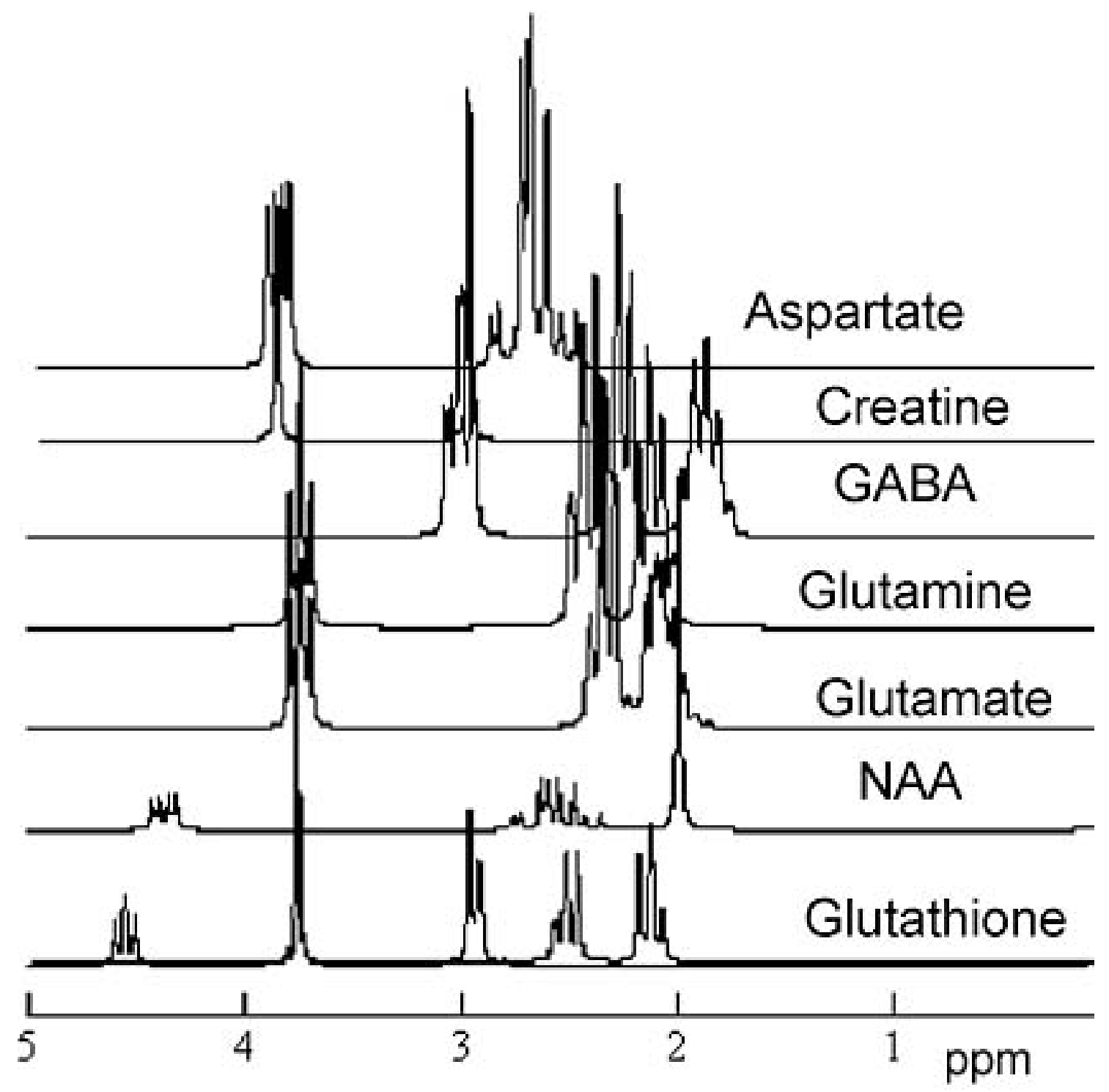

Figure 3.6: Simulated spectrum of various metabolites at 3.0T

Different versions of localized 2D sequences have been implemented by a number of other researchers. A COSY spectrum produces better dispersion of J-cross-peaks than a 2D J-resolved spectrum. Recently a localized version of COSY (L-COSY) has been developed by Thomas et al[26]. All the protons in the metabolite create diagonal peaks, while the coupled protons create cross peaks. Due to the dispersive nature of cross peaks, it may not be possible to observe the cross peaks that are close 
to the diagonal. A double quantum filtered COSY will greatly simplify the resulting spectra, and will help in quantification.

\subsubsection{Interpreting the 2D Spectrum}

Conventional NMR spectra (one-dimensional spectra) are plots of intensity vs frequency. In two dimensional spectroscopy the signal intensity is plotted as a function of two frequencies $F_{1}$ and $F_{2}$. The most common way of representing a 2D spectrum, is by using a contour plot in which the intensities of peaks are represented by contour lines drawn at suitable intervals. The position of each peak is specified by two frequency co-ordinates corresponding to $F_{1}$ and $F_{2}$. Two dimensional spectra are arranged so that the $F_{2}$ co-ordinates of the peaks correspond to the one-dimensional spectrum.

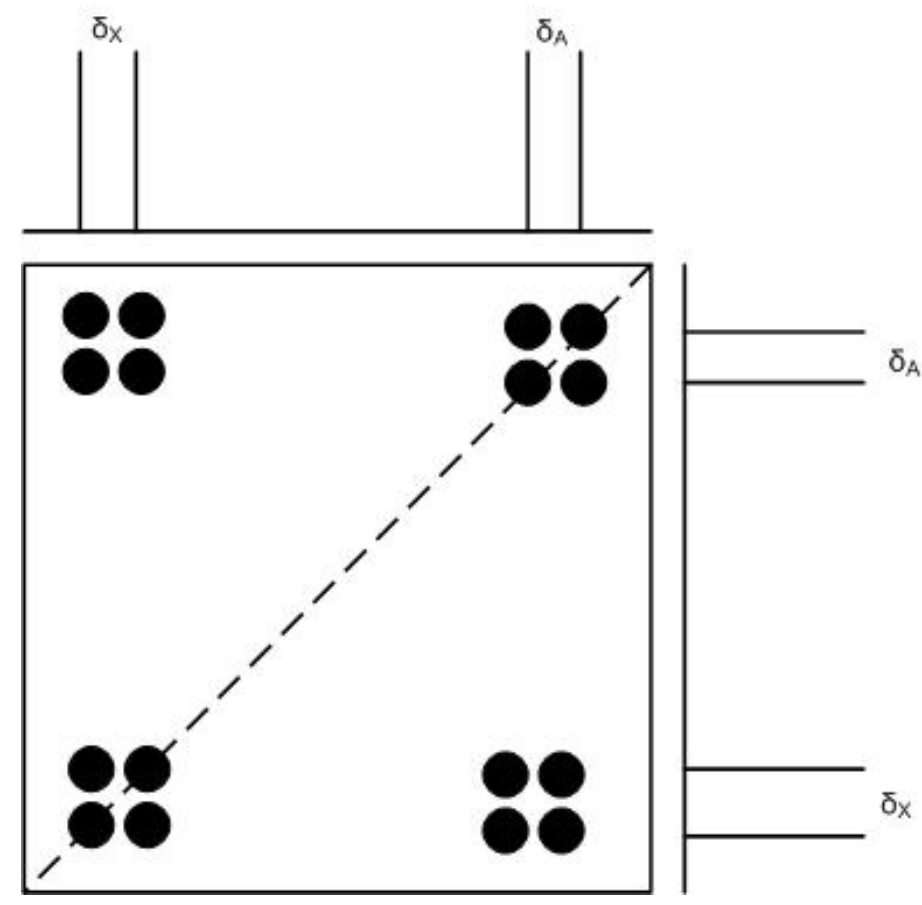

Figure 3.7: Schematic COSY spectrum

Fig. 3.7 shows a schematic of a COSY spectrum of a hypothetical molecule containing just two protons, $\mathrm{A}$ and $\mathrm{X}$ which are coupled to each other. The one- 
dimensional spectrum is plotted along the $F_{2}$ axis, and consists of pair of doublets centered on the chemical shifts of $A$ and $X, \delta_{A}$ and $\delta_{B}$ respectively. In the COSY spectrum, the $F_{1}$ co-ordinates of the peaks in the two-dimensional spectrum also correspond to those found in the normal one-dimensional spectrum. The COSY spectrum has symmetry about the diagonal $F_{1}=F_{2}$, and is marked by a dotted line.

In a $1 \mathrm{D}$ spectrum, scalar couplings give rise to multiplets in the $1 \mathrm{D}$ spectrum. In two-dimensional spectra, a multiplet consists of an array of individual peaks with a square or a rectangular outline. Such peaks are shown in the schematic COSY spectrum for two coupled spins A and X. These two-dimensional multiplets can be classified as diagonal peaks, which appear at the same $F_{1}$ and $F_{2}$ frequency coordinates and cross peaks, which appear at different $F_{1}$ and $F_{2}$ frequency co-ordinates. In the schematic COSY spectrum, there are two diagonal multiplets, centered at $F_{1}=F_{2}=\delta_{A}$ and $F_{1}=F_{2}=\delta_{X}$, one cross-peak centered at $F_{1}=\delta_{A}, F_{2}=\delta_{X}$ and the other cross-peak centered at $F_{1}=\delta_{X}, F_{2}=\delta_{A}$. The cross-peak multiplet at $F_{1}=\delta_{A}, F_{2}=\delta_{X}$ in the COSY spectrum signifies that the two protons at chemical shifts $\delta_{a}$ and $\delta_{X}$ have a scalar (spin-spin) coupling between them.

If a peak appears at $F_{1}=\delta_{1}, F_{2}=\delta_{2}$, it means that the signal evolved at $\delta_{1} H z$ during $t_{1}$ and during the mixing period the coherence was transferred to another coherence precessing at $\delta_{2} H z$. If $\delta_{1}=\delta_{2}$, the coherence was unaffected during the mixing period. If there are two peaks appearing, one at $F_{1}=F_{2}=\delta_{1}$ and another at $F_{1}=\delta_{1}, F_{2}=\delta_{2}$, coherence was initially precessing at $\delta_{1} H z$ before the mixing period. During the mixing period part, the coherence has been transferred to $\delta_{2}$ and hence the two peaks. We see that the mixing time plays a very important role in generating the two-dimensional spectrum. 


\section{Chapter 4}

\section{Multiple Quantum Coherences}

This chapter describes the preparation, detection and properties of multiple quantum coherences. The energy level of a two-spin system is shown in Fig. 4.1. There are four single quantum transitions, marked by the dotted lines. The transition from $\alpha \alpha$ to $\beta \beta$ is called as a double quantum transition, as it involves the flip of two spins. The vector model fails to model experiments involving multiple-quantum coherence. The product operator formalism is better suited for the analysis of MQC's.

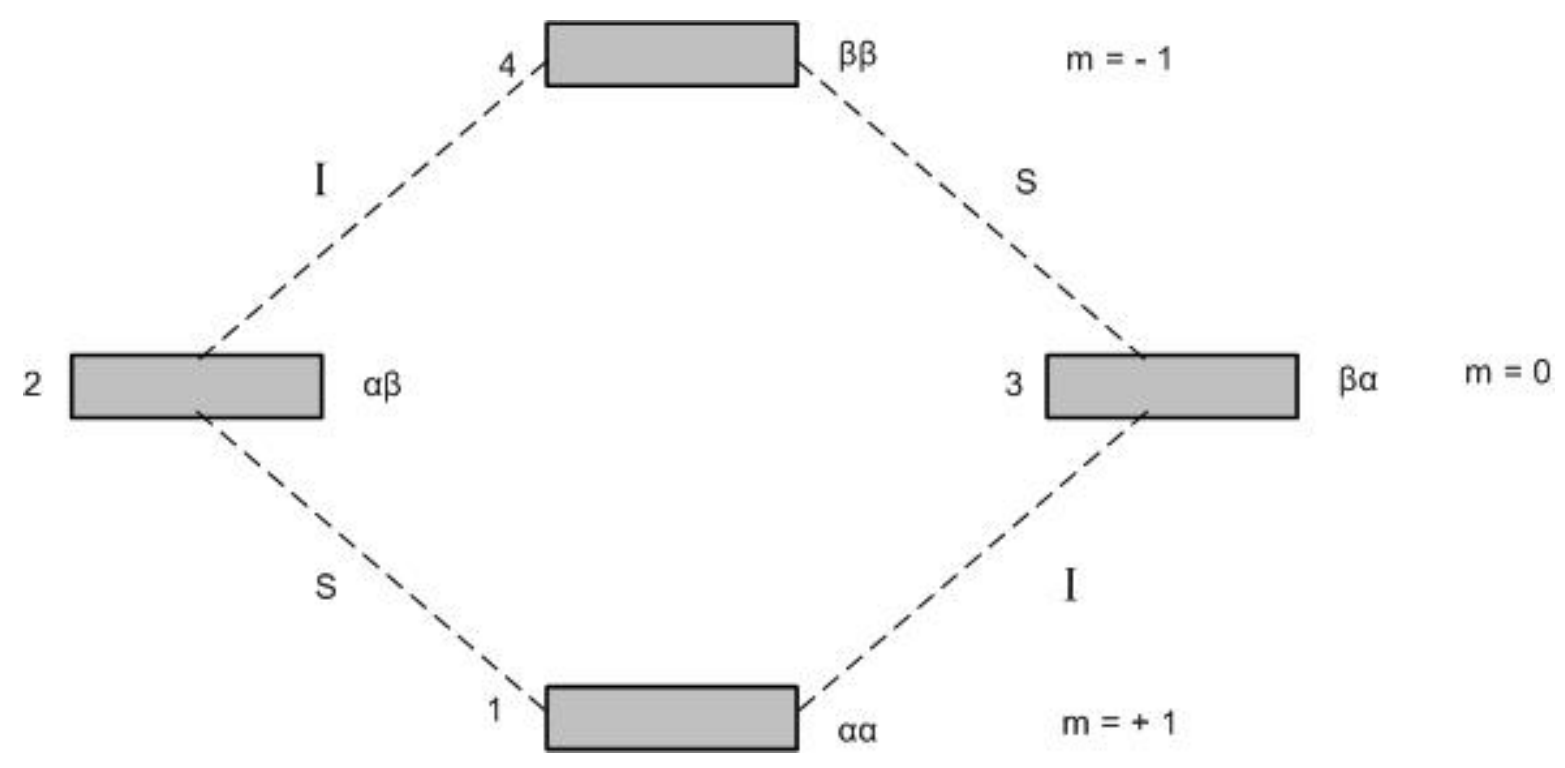

Figure 4.1: Energy level diagram of a coupled two spin system 


\subsection{Product Operator Formalism}

The vector model is based firmly on geometry, the product operator treatment mainly relies on algebra. The state of a spin system can be described by the density operator $\sigma(t)$. The time evolution of this density matrix can be determined for a given MR pulse sequence.

$$
\frac{d \sigma(t)}{d t}=-i|H(t), \sigma(t)|
$$

where $H$ is the Hamiltonian.

For simplicity we consider only weakly-coupled spin $+\frac{1}{2}$ nuclei, and neglect relaxation. The NMR experiment can be broken into a sequence of consecutive time intervals. Within each interval we may consider a cascade of evolutions due to chemical shift and spin-spin coupling, or a RF pulse. The time dependence of the RF pulse is removed by transformation into the appropriate rotating frame of reference. A hard RF pulse may be expressed as a cascade of soft pulses applied to different spins in turn, in any order. An amplitude or phase modulated pulse is decomposed into a sequence of many segments, each of constant phase and amplitude. The processes occurring within any time given time time instant $k$ can be expressed as a propagator, $\exp \left(-\left|H_{k}\left(t_{k}\right)\right|\right)$, and the time evolution computed as a sequence of such propagators.

The observable absorption-mode signal is evaluated as

$$
M_{y}(t)=N \gamma(h / 2 \pi) \operatorname{Tr}\left[\sum_{k} I_{k y} \sigma(t)\right]
$$

where $N$ is the number of spins per unit volume.

The density operator can be expressed as a sum of linear combination of basis functions $\left(B_{s}\right)$

$$
\sigma(t)=\sum_{s} b_{s}(t) B_{s}
$$

It is important to choose the basis functions that retain the clearest physical insight. 
Using the Cartesian product operators [43]

$$
B_{s}=2^{(q-1)} \prod_{k=1}^{n}\left(I_{k v}\right)^{a_{s k}}
$$

Where $n$ is the total number of spins in the spin system under consideration, $k$ is an index for the spin, and $\nu$ represents the $x, y$ or $z$ axis. The spin operators are multiplied $q$ at a time $(0 \leq q \leq n)$. The exponent $a_{s k}$ is a device to define the spins involved in the product operator, but zero for the remaining $n-q$ spins. Thus for an $I S$ spin system where $n=2$, we consider $q=0,1$ and 2. For $q=0$, the operator is $+\frac{1}{2} E$ where $E$ is the unity operator. For $q=1$ we have the single spin operators

$$
I_{x}, I_{y}, I_{z}, S_{x}, S_{y}, S_{z}
$$

while for $q=2$ there are nine two-spin operators:

$$
\begin{array}{lll}
2 I_{x} S_{x} & 2 I_{x} S_{y} & 2 I_{x} S_{z} \\
2 I_{y} S_{x} & 2 I_{y} S_{y} & 2 I_{y} S_{z} \\
2 I_{z} S_{x} & 2 I_{z} S_{y} & 2 I_{z} S_{z}
\end{array}
$$

The product operators can be illustrated for a coupled two-spin system. For simplicity we assume that we are dealing with spin $+\frac{1}{2}$ nuclei and that the spins are weakly coupled.

\subsubsection{Spin Populations}

The operators $I_{z}$ and $S_{z}$ represent the magnetization of $I$ and $S$ spins respectively. The magnetizations are proportional to the relevant energy levels. At thermal equilibrium, the state of a homonuclear spin system would be represented as

$$
I_{z}+S_{z}
$$

This is proportional to the total $Z$ magnetization of each spin species. Relaxation effects are normally ignored in the product operator treatment. 


\subsubsection{Magnetization}

The operators $I_{y}$ and $S_{y}$ represent transverse magnetization aligned along the $+y$ axis of the rotating frame of reference. In the vector model of a coupled two-spin system, we would represent this situation by two I-spin vectors aligned along $+y$, corresponding to an absorption-mode signal. This would be the situation immediately after a $(\pi / 2)_{x}$ excitation pulse. In a similar manner, $I_{x}$ and $S_{x}$ indicate transverse magnetization along the $+x$ axis.

When the transverse magnetization $I_{y}$ is allowed to precess freely, the magnetization vectors associated with the two lines of the doublet diverge and eventually reach an antiphase configuration. If we ignore the chemical shifts evolution (which can be refocussed if desired), we may represent this state by $2 I_{x} S_{z}$. This is $x$ magnetization of the $I$ spin, antiphase to the $S$ spin. The tiny magnetic fields exerted by the $S$ spin at the site of the $I$ spin have induced a differential precession of the two vectors. A similar term stands for antiphase $I$ spin along the $\pm y$ axes.

Instead of the familiar in-phase structure $I_{y}$, these product operator terms generate an up-down pattern of intensities within the multiplet. The antiphase magnetization is a characteristic feature of many coherence transfer experiments. Note that antiphase magnetization induces no signal in the receiver coil, but it can evolve into an in-phase component that is detectable.

\subsubsection{Coherence}

The third type of product operator represents a coherent superposition of states usually called coherence. Though this concept applies to states separated by $\Delta m=$ \pm 1 , it is used to identify (unobservable) multiple-quantum features involving states separated by $\Delta m \neq \pm 1$.

The product operator formalism describes how multiple-quantum coherence can 
be excited, how it is affected by spectrometer parameters, and how it may be converted into observable magnetization. These product operator terms represent a concerted coherent motion of two or more spins. For the two-spin system there are four such terms $2 I_{x} S_{x}, 2 I_{x} S_{y}, 2 I_{y} S_{x}, 2 I_{y} S_{y}$. In homonuclear systems, linear combinations of these terms represent pure zero- or pure double-quantum coherence.

The raising and lowering operators changes the magnetic quantum number by one unit either up or down. For a homonuclear system they are defined as

$$
\begin{aligned}
& I^{+}=I_{x}+\imath I_{y} \\
& I^{-}=I_{x}-\imath I_{y}
\end{aligned}
$$

We have a similar expression using $S^{+}$and $S^{-}$for $S$ spins. Consequently we have

$$
\begin{gathered}
I_{x}=+\frac{1}{2}\left(I^{+}+I^{-}\right) \\
I_{y}=\frac{1}{2 \imath}\left(I^{+}-I^{-}\right)
\end{gathered}
$$

If we are dealing with a concerted absorption or emission of two quanta in the same sense then the process involves the product $I^{+} S^{+}$and $I^{-} S^{-}$. There are two orthogonal forms of double-quantum coherence.

$$
\begin{array}{r}
I^{+} S^{+}+I^{-} S^{-}=2 I_{x} S_{x}-2 I_{y} S_{y} \\
I^{+} S^{+}-I^{-} S^{-}=\imath\left\{2 I_{x} S_{y}+2 I_{y} S_{x}\right\}
\end{array}
$$

If the two spins flip in opposite sense, the relevant products of the raising and lowering operators are $I^{+} S^{-}$and $I^{-} S^{+}$

$$
\begin{array}{r}
I^{+} S^{-}+I^{-} S^{+}=2 I_{x} S_{x}+2 I_{y} S_{y} \\
I^{+} S^{-}-I^{-} S^{+}=\imath\left\{2 I_{y} S_{x}-2 I_{x} S_{y}\right\}
\end{array}
$$

Thus there are two orthogonal forms of zero-quantum coherence. 


\subsubsection{Evolution}

Rotation about the $x$ or $y$ axes, caused by RF pules, and rotations about the $z$ axis, caused by chemical shifts or spin-spin coupling, are expressed by propagators of the form $\exp \left(-\imath H_{1} \tau_{1}\right)$. By convention, a sequence of such rotation operators is written in a time-reversed order (the first operation on the right).

$$
\sigma(t+\tau)=\exp \left(-\imath \Omega \tau I_{z}\right) \sigma(t) \exp \left(+\imath \Omega \tau I_{z}\right)
$$

In shorthand notation this can be written as

$$
\sigma \stackrel{\left(\Omega \tau \tilde{I}_{z}\right)}{\longrightarrow} \sigma^{+}
$$

To avoid confusion, the rotation angle is written in parentheses and the evolution super with a tilde $\left(\tilde{I}_{z}\right)$ to distinguish it from an operator $\left(I_{z}\right)$ that describes the state of a spin system. If we are considering only the effect on transverse magnetization $I_{y}$, the result is

$$
I_{y}=\stackrel{(\Omega \tau)-\tilde{I}_{z}}{\longrightarrow} I_{y} \cos \Omega \tau+I_{x} \sin \Omega \tau
$$

\subsection{Multiple Quantum Coherence(MQC)}

Coherence may be defined as the superposition of states of a spin or group of spins with a coherence phase relationship; in terms of energy levels, a coherence exists between levels available to a spin or group of spins when the spin or group populates each level and the phases of the coefficients for the state functions of each level have a coherent relationship. Coherences are often termed transitions, because a spin can be thought of as making transitions between energy levels in order to populate both the levels.

Transitions between energy levels are represented by a change in spin quantum number,

$$
p=\Delta m_{i j}=m_{i}-m_{j}
$$


Here $p$, is called order of transition. Transitions are classified according to their order, so transitions with $p=0, \pm 1, \pm 2$, will be called zero, single and double quantum transitions respectively. It is not possible to observe transitions with $p \neq \pm 1$ directly with simple single-pulse excitation. The transitions with $p> \pm 1$ are called multiple quantum transitions.

\subsection{Creating Multiple Quantum Coherence(MQC)}

A single non-selective pulse applied to a spin-system in thermal equilibrium will create single quantum coherence only [44]. When a pulse is applied to a spin-system that is in a non-equilibrium state, multiple quantum coherences $[45\rfloor$ are formed. Hence the number of ways of creating multiple quantum coherence is infinite. The pulse sequences fall into three categories.

\subsection{1 $\left(\frac{\pi}{2}\right)_{\phi}-\frac{\tau}{2}-(\pi)_{\phi}-\frac{\tau}{2}-\left(\frac{\pi}{2}\right)_{\phi+\psi}$ excitation}

The standard three pulse sandwich shown in Fig. 4.2, can be employed for excitation of multiple quantum coherences. The multiple quantum preparation sandwich consists of $90^{\circ}-\frac{\tau}{2}-180^{\circ}-\frac{\tau}{2}-90^{\circ} \mathrm{RF}$ pulse sequence. The period $\tau$ can be optimized for maximizing the desired multiple quantum coherence. If the second $90^{\circ}$ pulse is in phase or $180^{\circ}$ out of phase with the first $90^{\circ}$ pulse, even order MQC's can be created. If the second $90^{\circ}$ pulse, is in quadrature with the first $90^{\circ}$ pulse, odd order coherences will be created. The advantage of this sequence is that the chemical shifts and any $B_{0}$ inhomogeneity will be refocused due to the $180^{\circ}$ pulse.

\subsection{2 $\left(\frac{\pi}{2}\right)_{x}-\tau-\left(\frac{\pi}{2}\right)_{x}$ excitation}

The most common way to excite multiple quantum coherence is the application of two non-selective $\left(\frac{\pi}{2}\right)_{x}$ pulses separated by a delay $\tau$. Such a sequence is shown in Fig. 4.3. The transverse magnetization created by the first pulse is redistributed by 


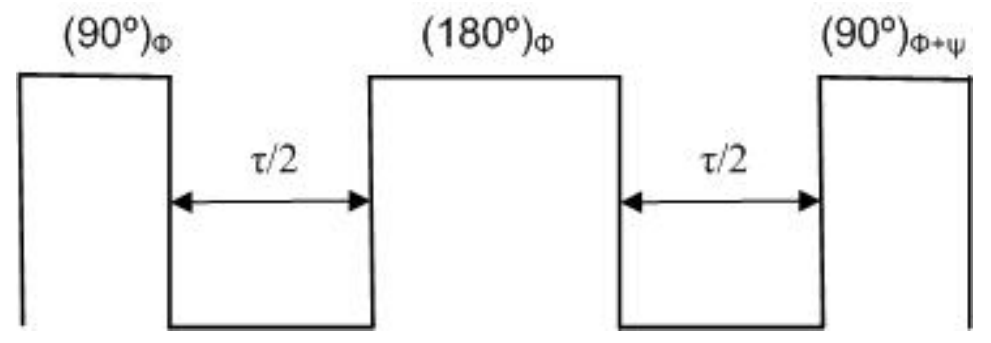

Figure 4.2: Three pulse sandwich for excitation of multiple quantum coherences

the second pulse over all possible transitions in the spin system., including multiple quantum transitions. The major problem in using non-selective excitation is that without using a density matrix calculation, the intensities of the transitions are very hard to determine for any coupled spin system consisting of more than two spins. The

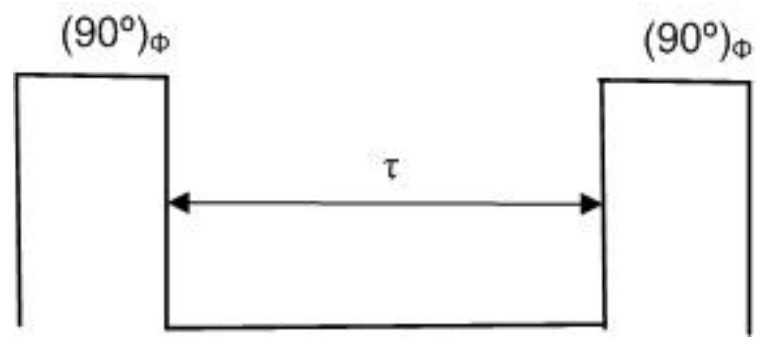

Figure 4.3: Two pulse sandwich for excitation of multiple quantum coherences

multiple quantum efficiency depends of the value of $\tau$. For $\tau=0$ the two $\left(\frac{\pi}{2}\right)_{x}$ pulses would just have the effect of a single $(\pi)_{x}$ pulse, not creating any multiple quantum coherences at all. The condition for excitation of a double or zero quantum coherence in which two spins $A$ and $X$ are the only spins participating is that $\sin \pi J_{A X} \tau \neq$ 0 . For a two spin example, it follows that maximum excitation can be achieved if $\sin \pi J_{A X} \tau=1$

\subsection{Detection of Multiple Quantum Coherences}

Multiple quantum coherences cannot be detected directly. The effects of multiple quantum coherences can be determined by applying an RF pulse after a time delay. This RF pulse transfers part of the multiple quantum magnetization to observable 
single quantum magnetization. A non-selective $\frac{\pi}{2}$ pulse is applied at a time $t_{1}$ after the multiple quantum is created. The coherence is then redistributed over all the transients present in the spin system. The magnetization components after the detection pulse are amplitude modulated as a function of $t_{1}$ by all the frequencies present in the spin system during the evolution period $t_{1}$. It is sufficient to detect the signal at a single point after a suitable time delay, since it contains information about the total vector sum of all transverse magnetization components which were modulated with the same frequencies. The modulation frequencies can be determined by the Fourier transform of the amplitude modulation envelope with respect to $t_{1}$.

\subsubsection{Single Point Acquisition}

A non selective-pulse cannot transfer multiple quantum magnetization into net transverse magnetization. The vector sum of the magnetization components, originating from multiple quantum coherence is zero, immediately after the second pulse. A time delay of $\tau$ is required before any signal is transferred from the multiple quantum, before the signal is acquired. This is used in the case when only one point is sampled for each value of $t_{1}$. To eliminate intensity distortions in the multiple quantum spectrum due to the fact that one value for $\tau$ will generally not be equally efficient for the observation of all different multiple quantum transitions, several experiments are performed for different values of $\tau$. The resulting absolute value mode multiple quantum spectra are then averaged to produce the final measurement.

\subsubsection{Multiple Point Acquisition}

The above procedure is inefficient and time consuming. Averaging over the different values of delay $\tau$ is automatically obtained if a two-dimensional experiment is performed where the signal after the detection pulse is collected over a period $t_{2}$. Intensity anomalies will only depend on the choice of the delay $\tau$ and will be significantly less severe. However, the number of data points collected will be higher. Since a non-selective pulse does not create any net magnetization, and magnetization 
components have opposite phases at $t_{2}=0$, the intensities of resonance lines in the two-dimensional phase-sensitive multiple quantum spectrum will either be positive or negative, in such a way that the total intensity integral over the spectrum is equal to zero. For this reason the absolute value mode is commonly used in the display of multiple quantum spectra. Since a non-selective pulse creates amplitude modulation of the detected signal with respect to $t_{1}$, the positive and negative multiple quantum frequencies cannot be distinguished.

\subsection{Special Properties of Multiple Quantum Co- herences}

Multiple quantum coherences are sensitive to magnetic field strengths, transmitter offset frequencies and phases of RF pulses which are characteristic of their order $[45,46]$. The effect of an extra offset $\Delta \omega$ on the frequency of coherence of order $p$ is a change by $p \Delta \omega$. The effect of a phase shift $\phi$ of the excitation pulses, on coherence of order $p$ is to produce a change in phase by $p \phi$. Both effects are incorporated in the formula

$$
\exp \left(\imath \phi F_{z}\right) \sigma_{p}\left(-\imath \phi F_{z}\right)=\exp (\imath p \phi) \sigma_{p}
$$

where $\sigma_{p}$ represents a density matrix only containing p-quantum elements $\sigma_{k l}$ with $M_{k}-M_{l}=p$ and the normal density matrix $\sigma=\sum_{p=-N}^{N} \sigma_{p}$, where $N$ is the number of spins in the system. More explicitly the effect on a matrix element $\sigma_{k l}\left(t_{1}\right)$ describing coherence between state $k$ and $l$ at time $t_{1}$ is given by

$$
\exp \left(\imath \phi F_{z}\right) \sigma_{k l}\left(-\imath \phi F_{z}\right)=\sigma(0) \exp \left(\imath \phi\left(M_{k}-M_{l}\right)\right) \exp \left(\imath \omega_{k l} t_{1}\right)
$$

where $\sigma(0)$ is the density matrix at time $t_{1}=0$. The operator $\exp \left(\imath \phi F_{z}\right)$ denotes a rotation over an angle $\phi$ about the z-axis. This rotation can either be caused by a static magnetic field along the z-axis or by a phase change of the RF pulse. 


\subsection{Order Selective Detection}

The single pulse detection scheme transfers multiple quantum coherence to observable-single quantum coherence, thus making indirect detection possible. Experimentally separating the effects of different orders of coherence is a difficult problem. The detection process can affect all orders of coherence present during evolution time. These coherences can be transferred to single-quantum coherence such that the observed signal will be derived from all orders of coherence.

It is possible to filter out selectively all but the desired orders of coherence, which are then indirectly detected. The filtering process is possible because the order of a coherence determines it characteristic response to a spin system [44, 47, 48]. Filtering can be achieved by using static field gradients $[45,49,50, ?]$ or rotary echo formation in an inhomogeneous RF field [51]. It can also be achieved by combining spectra measured with the same pulse sequence but with an altered pattern of relative phase of each RF pulse, called phase cycling [45].

Methods that use static-field gradients or rotary echoes rely on the fact that the angular velocity of precession of a coherence is proportional to both the strength of the applied field and the order of a particular coherence. A pair of gradient pulses can be applied symmetrically about a mixing sequence to select a particular coherence order. The relative strength and duration of the gradient pulses determine which orders are detected and the parameters of each pulse can be modified independently to control the filtering process.

\subsection{Coherence Transfer Pathways}

The sequence of events in a pulsed NMR experiment can be represented in a "coherence transfer map" $[47,48\rfloor$ as shown in Fig. for a double quantum preparation and mixing sequence. The orders of coherence precess freely within the levels of the map, 
and pulses can produce transition between levels. A coherence-transfer pathway is represented by the route a coherence follows during an experiment to reach the final order. As can be seen from the picture, there is more than one pathway to reach the desired coherence.

The process of coherence-transfer pathway selection is effectively a filtering procedure. Only specific coherences which can exist in a given spin system will traverse a particular pathway. Spin systems with all observable subspectra can be eliminated, if they do not have the required coherences. In a complex spectrum containing many overlapping multiplets from spin systems, can be simplified by filtering through appropriate multiple quantum coherences. 


\section{Chapter 5}

\section{Development of Localized DQF-COSY}

\subsection{Motivation for development of the DQF Lo- calized MRS}

The conventional COSY experiment suffers from a disadvantage which arises from the different phase properties of cross and diagonal-peak multiplets. The components of a diagonal peak mutliplet are all in-phase and so they tend to reinforce each other. The result of this reinforcement is the a broad intense signal which can obscure the nearby cross peaks. The dispersive tails of these peaks spread far into the spectrum. This effect is particularly troublesome when coupling is comparable with the line width. In addition to the above effects, the cancellation of anti-phase components in the cross-peak multiplets reduces the overall intensity of these multiplets. The signal from un-coupled spin systems such as water, methyl groups of NAA or creatine which are responsible for the overwhelming diagonal peaks can be suppressed, leading to a cleaner spectrum. This can be achieved by using a localized Double Quantum Filtered COSY sequence. 


\subsection{Design of the Localized DQF Sequence}

The pulse sequence is designed using three slice-selective $\mathrm{RF}$ pulses $\left(90^{\circ}-90^{\circ}-90^{\circ}\right)$ which serve the purpose of volume localization as well as creating the multiple quantum filter, in conjunction with gradients. The time delay between first two $90^{\circ}$ pulses is given by $t_{1}$. This time controls the mixing of the coherences. The second and third $90^{\circ}$ pulses are separated by a small time delay about $4 \mu S$.

The first two $90^{\circ}$ pulses create multiple quantum coherences as discussed in section 4.3.2. The third $90^{\circ}$ pulse acts as a coherence transfer pulse, converting the multiple quantum coherence into single quantum coherence for detection. The desired coherence pathway can be selected by using phase cycling $[52,53]$ in which the second $90^{\circ}$ pulse is phase cycled to retain only the coherences which are in multiple quantum coherence state after the second $90^{\circ}$ pulse. The disadvantage of this phase cycling approach is that it requires a four step phase cycle, of which only two of them yield cross peaks which are of interest, while the other two scans yield diagonal peaks which are cancelled out. The other option of coherence selection is by the means of gradients [54]. The slice select gradient introduces a phase shift to the different coherences. The negative going gradients symmetrically placed around the slice select gradients, are used to cancel the phase shift introduced by the slice select gradient.

\subsection{Theory of Localized DQF}

A theoretical analysis to calculate the amplitudes and phase of DQF-COSY cross and diagonal peaks is presented here. Here a two pulse system (IS) is considered as an example. To simplify the discussion one of the two spins is considered. At equilibrium we have $I_{z}+S_{z}$. Applying a $\frac{\pi}{2}$ pulse on $I_{z}$ results in

$$
I_{z} \stackrel{\frac{\pi}{2} I_{x}}{\longrightarrow} \stackrel{\frac{\pi}{2} S_{x}}{\longrightarrow}-I_{y}
$$


This state evolves for the time $t_{1}$, first under the influence of frequency offset of spin 1. Offset of spin 2 does not have any effect on spin 1 operators.

$$
-I_{y} \stackrel{\omega_{1} t_{1} I_{z}}{\longrightarrow}-\cos \omega_{1} t_{1} I_{y}+\sin \omega_{1} t_{1} I_{x}
$$

Both the terms in Equation 5.2 then evolve under the scalar coupling.

$$
\begin{array}{r}
-\cos \omega_{1} t_{1} I_{y} \stackrel{2 \pi J_{12} I_{z} S_{z}}{\longrightarrow}-\cos 2 \pi J_{12} t_{1} \cos \omega_{1} t_{1} I_{y} \\
+\sin \pi J_{12} t_{1} \cos \omega_{1} t_{1} 2 I_{x} S_{z} \\
\sin \omega_{1} t_{1} I_{x} \stackrel{2 \pi J_{12} I_{z} S_{z}}{\longrightarrow}-\cos 2 \pi J_{12} t_{1} \sin \omega_{1} t_{1} I_{x} \\
+\sin \pi J_{12} t_{1} \sin \omega_{1} t_{1} 2 I_{y} S_{z}
\end{array}
$$

Equation 5.3 completes the evolution under the time $t_{1}$. Now we consider the effect of the second $90^{\circ} \mathrm{RF}$ pulse. We need to consider the effect of the pulse on both the spins.

$$
\begin{aligned}
& -\cos 2 \pi J_{12} t_{1} \cos \omega_{1} t_{1} I_{y} \stackrel{\frac{\pi}{2} I_{x}}{\longrightarrow} \stackrel{\frac{\pi}{2} S_{x}}{\longrightarrow}-\cos 2 \pi J_{12} t_{1} \cos \omega_{1} t_{1} I_{z} \\
& \sin \pi J_{12} t_{1} \cos \omega_{1} t_{1} 2 I_{x} S_{z} \stackrel{\frac{\pi}{2} I_{x}}{\longrightarrow} \stackrel{\frac{\pi}{2} S_{x}}{\longrightarrow}-\sin \pi J_{12} t_{1} \cos \omega_{1} t_{1} 2 I_{x} S_{y} \\
& -\cos 2 \pi J_{12} t_{1} \sin \omega_{1} t_{1} I_{x} \stackrel{\frac{\pi}{2} I_{x}}{\longrightarrow} \stackrel{\frac{\pi}{2} S_{x}}{\longrightarrow}-\cos 2 \pi J_{12} t_{1} \sin \omega_{1} t_{1} I_{x} \\
& \sin \pi J_{12} t_{1} \sin \omega_{1} t_{1} 2 I_{y} S_{z} \stackrel{\frac{\pi}{2} I_{x}}{\longrightarrow} \stackrel{\frac{\pi}{2} S_{x}}{\longrightarrow} \sin \pi J_{12} t_{1} \sin \omega_{1} t_{1} 2 I_{z} S_{y}
\end{aligned}
$$

The terms in Equation 5.5 are unobservable because the magnetization is in the $z$ direction. The term in Equation 5.6 contains the double quantum coherence. The terms in Equation 5.7 correspond to inphase magnetization along the $x$ axis. The terms in Equation 5.8 show the antiphase magnetization of spin $I$.

We can show that the terms in Equation 5.6 are indeed double quantum coherence by using the raising and lowering operators defined in section 4.1.3.

$$
\begin{aligned}
& 2 I_{x} S_{y}=2 \frac{1}{2}\left(I^{+}+I^{-}\right) \frac{1}{2 \imath}\left(S^{+}-S^{-}\right) \\
& 2 I_{x} S_{y}=\frac{1}{2 \imath}\left(I^{+} S^{+}-I^{-} S^{-}\right)+\frac{1}{2 \imath}\left(-I^{+} S^{-}+I^{-} S^{+}\right)
\end{aligned}
$$

The right side of Equation 5.10 contains both double and zero quantum coherence. The pure double quantum part is the term in the first bracket. This term can be 
reexpressed in the Cartesian operators as

$$
\begin{aligned}
\frac{1}{2 \imath}\left(I^{+} S^{+}-I^{-} S^{-}\right) & =\frac{1}{2 \imath}\left[\left(I_{x}+\imath I_{y}\right)\left(S_{x}+\imath S_{y}\right)-\left(I_{x}-\imath I_{y}\right)\left(S_{x}-\imath S_{y}\right)\right. \\
\frac{1}{2 \imath}\left(I^{+} S^{+}-I^{-} S^{-}\right) & =\frac{1}{2}\left[2 I_{x} S_{y}+2 I_{y} S_{x}\right]
\end{aligned}
$$

The effect of the last $90^{\circ}$ pulse on the double quantum part of Equation 5.6 is given by

$$
\begin{array}{r}
-\frac{1}{2} \sin \pi J_{12} t_{1} \cos \omega_{1} t_{1}\left(2 I_{x} S_{y}+2 I_{y} S_{x}\right) \stackrel{\text { 皿 } I_{x}}{\longrightarrow} \stackrel{\frac{\pi}{2} S_{x}}{\longrightarrow} \\
-\frac{1}{2} \sin \pi J_{12} t_{1} \cos \omega_{1} t_{1}\left(2 I_{x} S_{z}+2 I_{z} S_{x}\right)
\end{array}
$$

The first term on the right in Equation 5.3 is anti-phase magnetization of spin I aligned along the $x$-axis. This gives rise to a diagonal peak multiplet. The second term is anti-phase magnetization of spin $\mathrm{S}$ aligned along the $x$-axis. This will give rise to a cross-peak multiplet. Both of these terms have the same modulation in $t_{1}$, which can be shown from a similar analysis leading to an anti-phase multiplet along $F_{1}$ dimension. All these peaks have the same line shape. The overall phase of the spectrum can be adjusted so that they are all in absorption. This is in contrast to COSY, where the diagonal peaks are in-phase.

\subsection{Environment for Pulse Programming in $\mathrm{C}$ (EPIC)}

EPIC $\lfloor 1\rfloor$ is a programming language, a development environment, and a runtime environment for MR pulse sequences. A pulse sequence (or just a sequence) is a combination of linear orthogonal gradients (X, Y, Z), RF excitation and data collection that generates an MR image or MR data. It is typically an excitation with a certain phase encoding value followed by a data acquisition. An EPIC program is executed on a MR Scanner, and controls system hardware to generate such a sequence.

The process of compiling and linking the software EPIC programmer produces two executable files. The first executable is referred to as the PSD (Pulse Sequence 
Database) host process and is responsible for turning the operatorentered protocol information ( TE, TR, FOV etc.) into pulse parameters ( pulse widths, amplitudes, delays etc.) which are used by the second executable. The second executable is referred to as the PSD IPG (Integrated Pulse Generator) process and is responsible for turning the pulse parameters into a digital representation of a pulse sequence and then making the necessary real time modifications as the sequence is played out by the hardware.

The PSD source code is preprocessed and split into 2 source modules. An EPIC source file produces two resultant $\mathrm{C}$ source files, which are named filename.host.c and filename.ipg.c. The EPIC language provides compiler controls to aid in this splitting and to allow variables defined in the host section of code to be referenced in the IPG section.

\subsubsection{Interprocess Communication in EPIC}

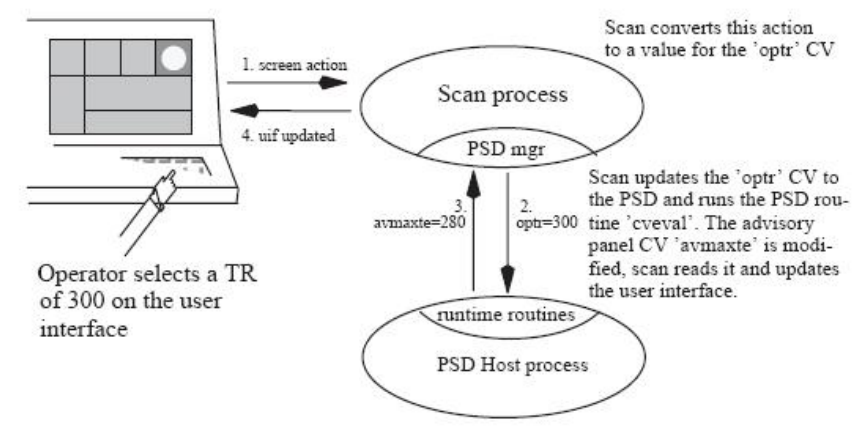

Figure 5.1: Interprocess Communication in EPIC. Reproduced from $[1]$

The communication is bidirectional. The Scan, PSD Host and PSD IPG processes are compiled with a core CV include file to allow the PSD manager to communicate the prescription via predefined variables. The interprocess communication is shown in Fig 5.1. 


\subsection{Implementing the Sequence in EPIC}

The DQF COSY sequence can be divided into three sections. Water suppression and spatial saturation (outer volume suppression) modules have been borrowed from the STEAMCSI pulse sequence provided with the GE 3T Horizon LX Scanner. Numerically optimized SLR Pulses $\lfloor 28,29,30,31,32,33\rfloor$ with durations of $3.6 m s$ or $1.8 m s$ depending on the choice of user. The time between the first two RF pulses can be adjusted by changing the opte $\mathrm{CV}$ to the required value. The time between individual gradients and slice select gradients can be adjusted by varying the delta1, delta2 and delta3 values.

\begin{tabular}{|c|c|c|c|c|}
\hline Axial & $\mathrm{A} / \mathrm{P}$ & $\mathrm{Y}$ & $\mathrm{X}$ & $\mathrm{Z}$ \\
\hline Axial & $\mathrm{R} / \mathrm{L}$ & $\mathrm{X}$ & $\mathrm{Y}$ & $\mathrm{Z}$ \\
\hline Coronal & $\mathrm{S} / \mathrm{I}$ & $\mathrm{Y}$ & $\mathrm{Z}$ & $\mathrm{X}$ \\
\hline Coronal & $\mathrm{R} / \mathrm{L}$ & $\mathrm{X}$ & $\mathrm{Z}$ & $\mathrm{Y}$ \\
\hline Sagittal & $\mathrm{S} / \mathrm{I}$ & $\mathrm{Z}$ & $\mathrm{Y}$ & $\mathrm{X}$ \\
\hline Sagittal & $\mathrm{A} / \mathrm{P}$ & $\mathrm{Z}$ & $\mathrm{X}$ & $\mathrm{Y}$ \\
\hline
\end{tabular}

Table 5.1: Anatomical to logical-co-ordinate conversion table

The incrementable time delay $t_{1}$ for the generation of the second dimension is introduced to the right of the slice balancing gradient slbal_rf1right of the first $90^{\circ}(r f 1)$ pulse. The slice balancing gradients such as (slbal_rf2left, slbal_rf2right) are used to compensate for the phase shift introduced by the slice select gradients (gzrf1, gxrf2 and gyrf3). The gradorder CV can be used to control the planes on which the RF pulses appear, and gives the relationship between the logical and patient co-ordinates. The anatomical to logical co-ordinate conversion table is given in 5.1.

The gradients gxc12, gyc23 and gyc34 amplitudes can be adjusted using the CV grad_ratio, so that the gradients are scaled to the required ratios. The different values of the CV, and the corresponding ratios of the gradients are given in Table 5.2.

The slbal_option CV can be used to turn the slice balancing gradients ON and OFF. These options were used during optimizing the sequence for maximum signal. 


\begin{tabular}{|c|c|c|c|}
\hline grad_ratio & area of crusher gxc12 & area of crusher gxc23 & area of crusher gxc3r \\
\hline 2 & 4 & 3 & 10 \\
\hline 4 & 0 & 0 & 0 \\
\hline 7 & 0 & 1 & 2 \\
\hline 8 & 0 & 1 & -2 \\
\hline
\end{tabular}

Table 5.2: Gradient ratios for coherence pathway selection

The grad_scale CV can be used to scale the areas of crusher gradients, so that they do not swamp the entire magnetization. These options proved to be helpful, when the sequence was optimized for maximum signal intensity.

The options $4: 3: 10,0: 1: 2$ and $0: 1:-2$ permits selection of the doublequantum to single-quantum pathway. The option $0: 0: 0$ permits to detect the single quantum signal in the absence of pathway selection gradients. The programming includes a provision to vary the gradient areas by $1 \%$ which permits optimal balancing of the pathway selection gradients. 


\section{Chapter 6}

\section{Experimental Results}

In this chapter we demonstrate the localized double-quantum-filtered COSY technique on simple molecules and also in vivo on human calf muscle. We demonstrate the efficiency of filtering the single quantum coherences by performing L-COSY on the same molecules, which excites and detects single quantum coherences.

\subsection{Optimization}

\subsubsection{Optimizing Water Suppression}

The water suppression and outervolume suppression modules in the DQF-COSY sequence have been borrowed from the STEAMCSI pulse sequence provided with the GE 3T Horizon LX System. The Automatic Water Suppression(AWS) process is part of the Autoprescan module. The AWS sequence has been designed by GE to be somewhat insensitive to variations in $T 1$ and $B 1$. The AWS process adjusts the flip angle (power) of the third water suppression RF pulse. However these flip angles might not be optimum for the subject. They can be optimized by executing the manual prescan module, and observing the resultant 1D spectrum. The flip angles of the RF pulses used in the water and outervolume suppression modules can be changed on the fly, and the resultant spectrum is updated automatically. The best possible values can be found by trial and error. The autoprescan module also determines the receive and tranmit gains $(R 1, R 2$ and $T G)$, and the center frequency. 


\subsubsection{Shimming}

First order and higher order shimming are performed on the subject to get a uniform $B_{0}$ field. This is very important as inhomogeneities in the $B_{0}$ field will affect the frequency of precession and hence the resultant spectrum. The gradient shimming control panel allows the user to adjust the linear $x, y$ and $z$ shims by adjusting the corresponding gradient currents.

\subsubsection{Optimizing crusher gradients}

The spectroprescan module, provides a preview of the 1D spectrum after the application of the entire sequence. This includes water suppression, outervolume suppression and the DQF-COSY sequence. The gradient scaling factors can be adjusted here either in the presence or absence of water suppression. Water does not have any coupled spins and it should not pass through the double-quantum-pathway. This property can be used to adjust the crusher gradient ratios so that they are optimum for the pathway selection. The gradients areas are adjusted to get the smallest water peak as possible.

\subsection{Scan}

The various scan parameters need to be setup on the scan desktop to run the scan. The type of the coil (head coil, surface coil, quad-knee coil) that is being used should also be specified. The imaging parameters such as Plane, Mode, Pulse Seq, Imaging Options are to be set for the $3 P$ Localizer sequence. The $3 P$ Localizer sequence needs to be run before a spectroscopy experiment to provide the user with a graphical tool, to place the voxel over the VOI. The important scan CV's such as TE and TR need to be set before a scan can be initiated. The number of excitations over which each scan has to be averaged has to be set in the CV NEX. Phase cycling is not used in DQF-COSY, hence NEX can be set to any desired number. Generally the spectrum is averaged over 8 scans to give a better signal to noise ratio. 
The 3P Localizer sequence used to generate the pilot image was performed with the following parameters: $T R=2000 \mathrm{~ms}, T E=30 \mathrm{~ms}, F O V=15 \mathrm{~cm}, N E X=1$ and with 256 x 128 points in $F_{2}$ and $F_{1}$ dimensions respectively. The desired voxel of size $3 \times 3 \times 3 \mathrm{~cm}^{3}$ was selected from the pilot image, and the spectrum is collected from the selected voxel. The parameters used for DQF-COSY are $T R=2000 \mathrm{~ms}$, $T E=30 \mathrm{~ms}, F O V=15 \mathrm{~cm}, N E X=8$ and with $1024 \times 64$ points in $F_{2}$ and $F_{1}$ dimensions respectively. The 2D spectrum thus obtained is linear predicted to 1024 x 128 using Felix, a commercial NMR data processing tool. The resultant matrix is then zero filled to $4096 \times 256$ points and is $2 \mathrm{D}$ Fourier transformed to obtain the 2D spectrum.

\subsection{Post Processing Data}

The two dimensional raw data that is obtained from the scan cannot be processed by the tools provided by GE, as they can only be used for imaging or 1D spectroscopy data sets. Hence the data obtained is processed by a commercially available NMR tool called Felix.

\subsubsection{Apodization}

The signal to noise ratio (SNR) of the collected data can be improved by a data processing technique called apodization. Apodization is essentially multiplication of an FID by a window function. The function is computed for the time domain of interest and is multiplied point for point into time domain data. This results in the following advantages.

- Apodization to avoid truncation artifacts with zero filling

- Maximization of $\mathrm{S} / \mathrm{N}$ based on peak intensities.

- Maximization of resolution by minimizing line widths. 
- Conversion of the frequency-domain line shape into a more desirable form.

The principle of matched filtering is used here to maximize the SNR. The principle of matched filtering states that maximum $\mathrm{S} / \mathrm{N}$ is obtained when with a window function $w(t)$ matches the form of signal modulation envelope $s_{e}(t)$. Sinebell and Sinebell squared windowing functions are used in these experiments as they emphasize the sine-modulated peaks.

\subsubsection{Linear Prediction}

Linear prediction is the method of directly obtaining resonance frequencies and relaxation rates from time domain signals, which are the superposition of exponentials, by solving the characteristic polynomial. Phases and intensities must be calculated iteratively using a least-square procedure. In the presence of noise, the total number of exponentials assumed must be greater than the number of cross peaks expected. If the number of unknowns is $n$ and $m$ is the number of data points used for the prediction, the number of operations is proportional to $m n^{2}$. The computational complexity increases rapidly with the number of observed resonances. Most of the linear predictions are based on a reduction in dimensionality.

One of the four methods, viz. forward, backward, forward-backward and mirror image methods, may be used for linear prediction in Felix. All these techniques require that the number of coefficients to be used for prediction be specified. The forward-backward technique does not require any prior knowledge about the FID and is very robust. This is used in linear prediction of all the acquired data sets.

\subsection{Results and Discussion}

\subsubsection{Results on Ethanol Phantom}

We show the suppression of single quantum coherences, in a phantom containing a mixture of ethanol and water. Water can create only single quantum coherences, and 
is totally suppressed by the sequence. The phantom was prepared with the following compounds in a $250 \mathrm{ml}$ spherical flask.

- $225 \mathrm{ml}$ of laboratory grade ethanol $\left(\mathrm{CH}_{3} \mathrm{CH}_{2} \mathrm{OH}\right)$

- $25 \mathrm{ml}$ of water $\left(\mathrm{H}_{2} \mathrm{O}\right)$

Laboratory grade ethanol with $90 \%$ concentration has been used here. The chemical structure of ethanol is shown in Fig. 6.1

$$
\begin{aligned}
& \mathrm{OH}-\mathrm{CH}_{2}-\mathrm{CH}_{3} \\
& \begin{array}{lll}
\text { (C) } & \text { (B) }
\end{array}
\end{aligned}
$$

Figure 6.1: Chemical Structure of Ethanol

The 1D spectrum of ethanol is shown in Fig. 6.2. The protons in the methyl $\left(\mathrm{CH}_{3}\right)$

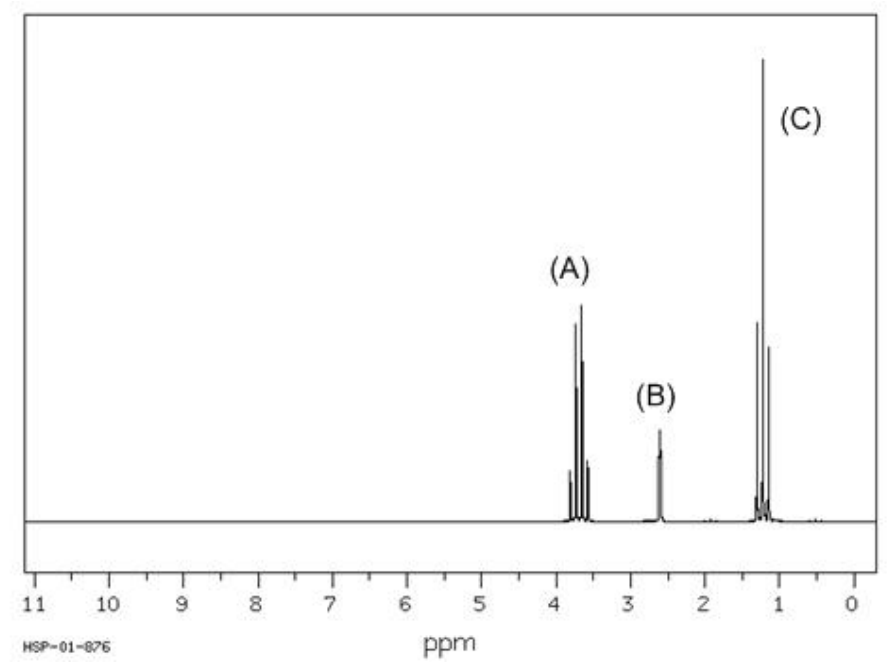

Figure 6.2: One dimensional spectrum of Ethanol Phantom

and methylene $\left(\mathrm{CH}_{2}\right)$ groups in ethanol are coupled to each other. The methyl group splits into a quartet and the methylene group splits into a triplet due to the scalar coupling between them. The protons in water are not coupled with any other protons, hence they are observed as a singlet. The L-COSY of the phantom is shown Fig. 6.3. 


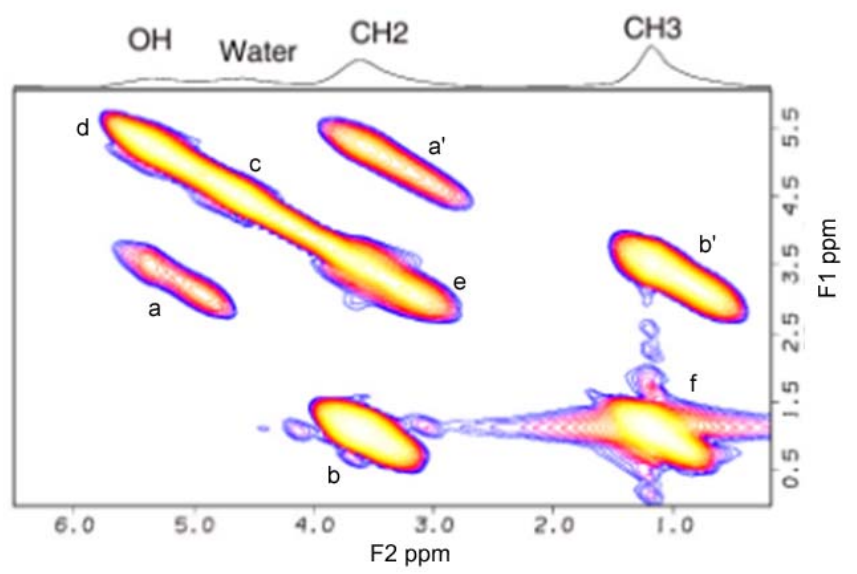

Figure 6.3: L-COSY spectrum of Ethanol Phantom

L-COSY excites and detects single quantum coherences only. It is clear from Fig 6.3 that all the single quantum coherences have been excited and detected. The cross peaks labelled $a$ and $a^{\prime}$ are due to the coupling between $\mathrm{OH}$ and $\mathrm{CH}_{2}, b$ and $b^{\prime}$ are due to the coupling between $\mathrm{CH}_{2}$ and $\mathrm{CH}_{3}$. The diagonal peaks: $c$ is due to water, $d$ is due to the $\mathrm{OH}$ group, $e$ is due to $\mathrm{CH}_{2}$ and $f$ is due to $\mathrm{CH}_{3}$.

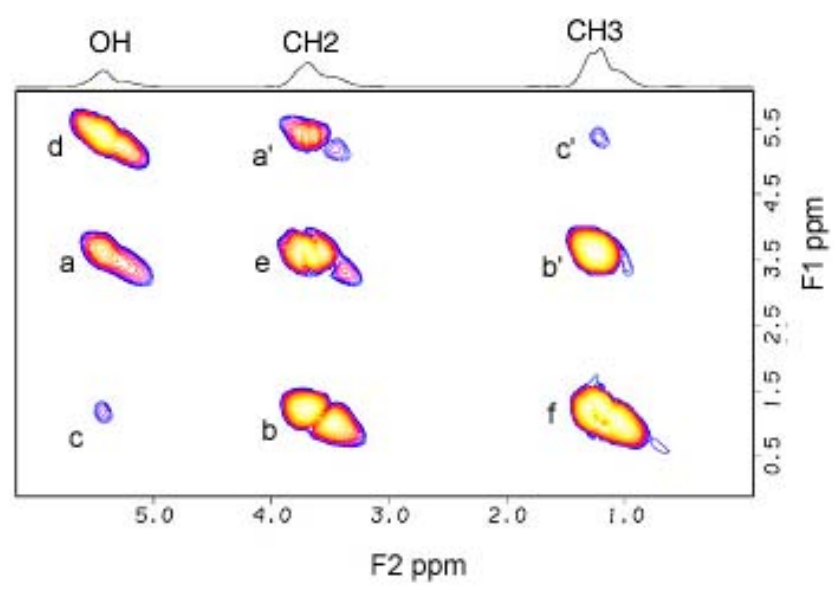

Figure 6.4: DQF-COSY spectrum of Ethanol Phantom

The DQF-COSY spectrum of the ethanol phantom is shown if Fig. 6.4. DQFCOSY excites double quantum and detects single quantum coherences. We see that 
the diagonal peak $c$ due to water has disappeared completely. All the other cross and diagonal peaks due to $\mathrm{CH}_{2}, \mathrm{CH}_{3}$ and $\mathrm{OH}$ are retained because they are coupled with each other. We also see that the diagonal peaks are not dispersive as in L-COSY because they are anti-phase coherences.

We also see a cross peak between the $\mathrm{CH}_{3}$ and $\mathrm{OH}$ group in the DQF-COSY spectrum. This might be due to additional pathways due to exchange pathways, which is not investigated any further in this thesis.

\subsubsection{Results on Ethyl Acetate}

The chemical structure of ethyl acetate is shown in Fig. 6.5.

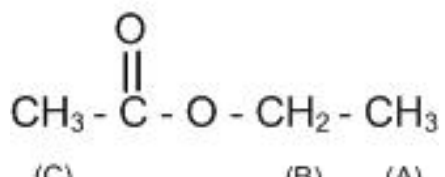

Figure 6.5: Chemical structure of ethyl acetate

The 1D spectrum of ethyl acetate obtained using a PRESS sequence is shown in Fig. 6.6. The protons in the methyl group $\left(\mathrm{CH}_{3}\right)$ labelled $A$ and methylene $\left(\mathrm{CH}_{2}\right)$ group labelled $B$ are coupled to each other and produces multiplets in the 1D spectrum. The protons in the methyl group labelled $C$ is not coupled to any other protons, and produces a singlet. The peaks are appropriately labelled in Fig. 6.6 The L-COSY spectrum of ethyl acetate is shown in Fig 6.7. The cross peaks due to coupling between the methyl $(A)$ and the methylene $(B)$ groups are marked as $a^{\prime}$. The diagonal peaks of $B, C$ and $A$ are marked $b, c$ and $d$ respectively.

The DQF-COSY spectrum of ethyl acetate is shown in Fig. 6.8. The protons in the methyl group labelled $C$ are not coupled with any other protons and hence can create only single quantum coherence. This signal is filtered by the double quantum pathway. This is clear from the Fig. 6.8. 


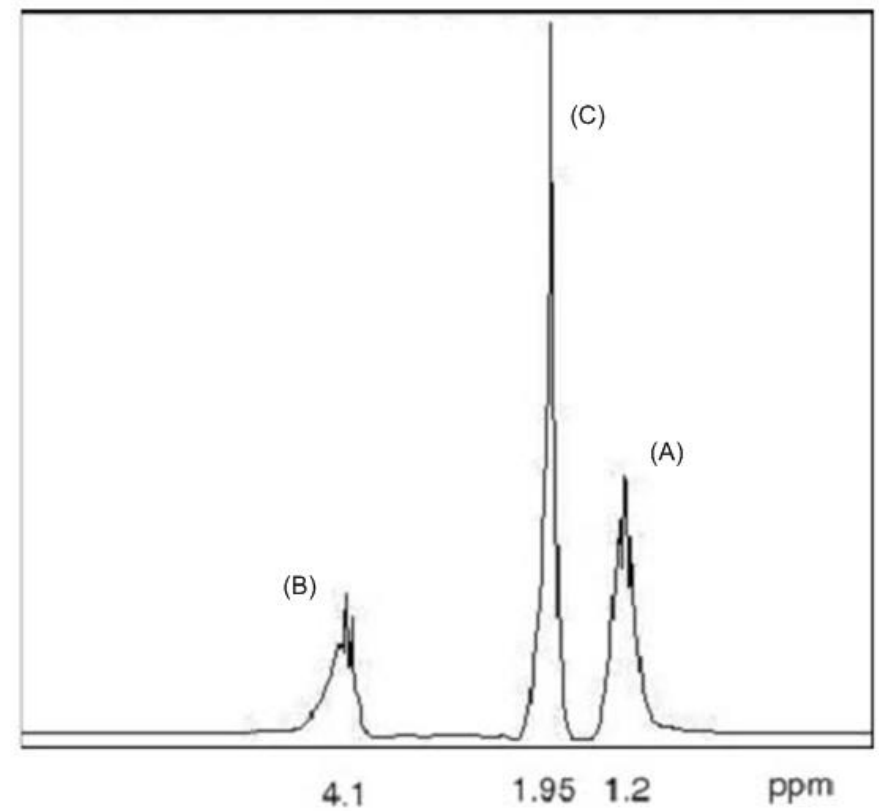

Figure 6.6: One-dimensional spectrum of ethyl acetate

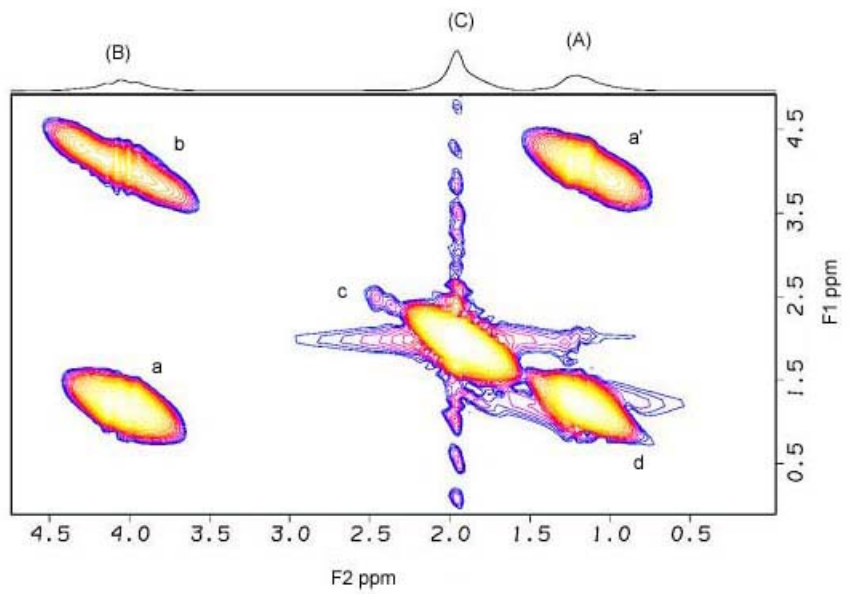

Figure 6.7: L-COSY spectrum of ethyl acetate

\subsubsection{In Vivo Results on Human Calf Muscle}

Myocellular lipids are important in the pathogenesis of insulin resistance. There are two two distinct compartments of lipids in the muscle. In one compartment lipids are contained within the fat cells, termed extramyocellular lipids (EMCL), and in other compartment intramyocellular lipids (IMCL) are located along with enzymes involved in fatty acid esterification, hydrolysis, and transport into the mitochondria 


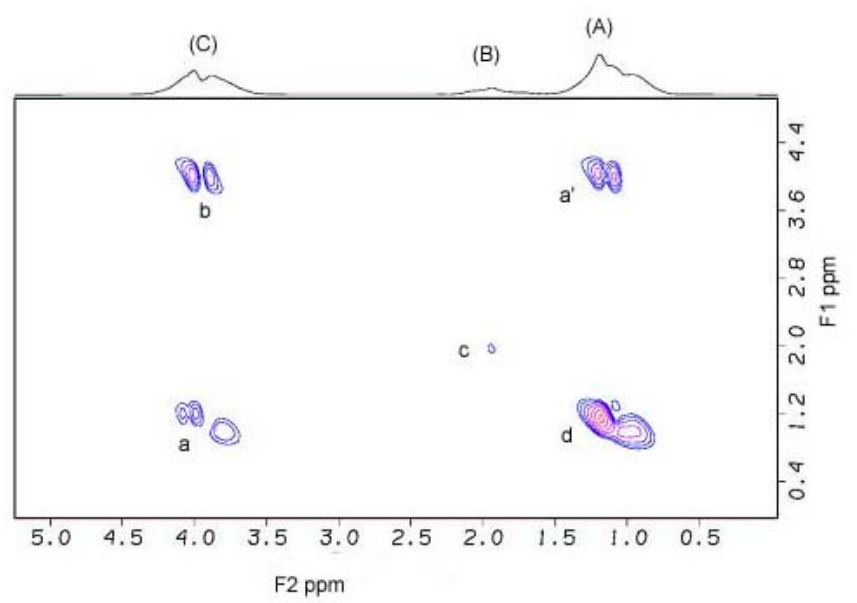

Figure 6.8: DQF-COSY spectrum of ethyl acetate with a lower threshold

which is closely associated with the cellular oxidation. Enhanced storage of IMCL occurs only under combined effects of high concentration serum insulin and free fatty acids. IMCL content of the muscle is correlated to glucose-insulin-lipid metabolism and insulin sensitivity.

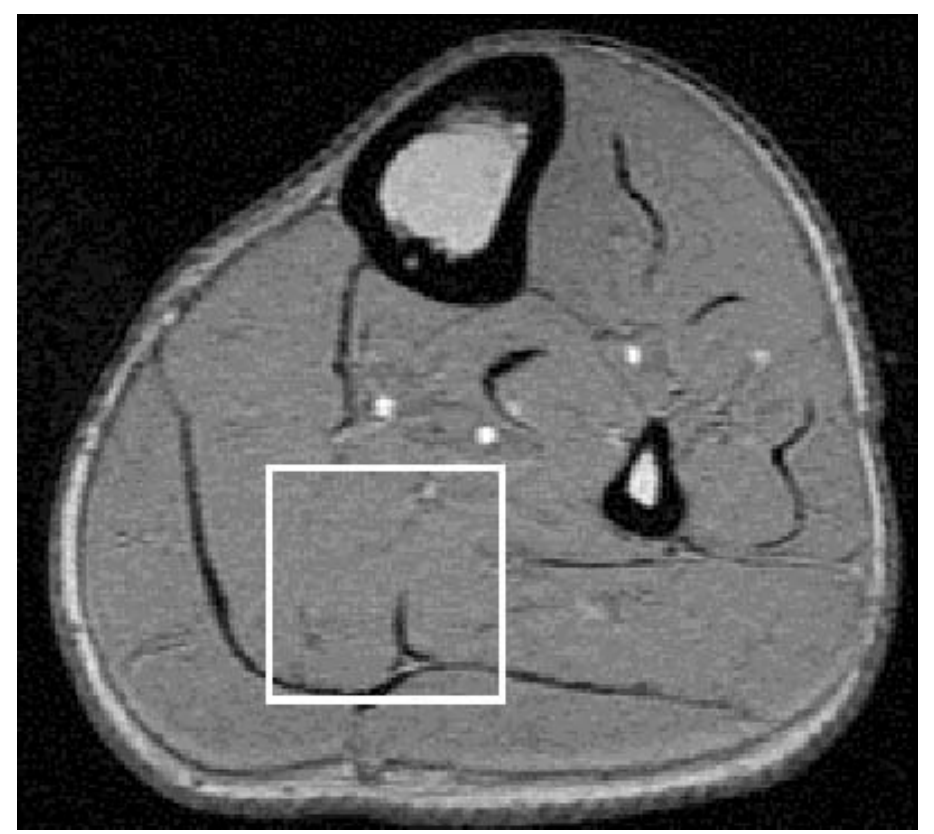

Figure 6.9: Calf muscle image

Fig. 6.9 shows the image of the muscle obtained from a 23 year healthy volunteer 
slice thickness of $5 \mathrm{~mm}$ and in-plane spatial resolution of $468 \mu \mathrm{m} \times 468 \mu \mathrm{m}$ acquired with a repetition time of $14.7 \mathrm{~ms}, 1$ average and echo time of $2.25 \mathrm{~ms}$. The location of the voxel selected for MRS studies is indicated in the image.

The one-dimensional spectra of calf muscle in shown in Fig 6.10. The peaks of EMCL and IMCL are heavily overlapping, and is extremely difficult to quantify them.

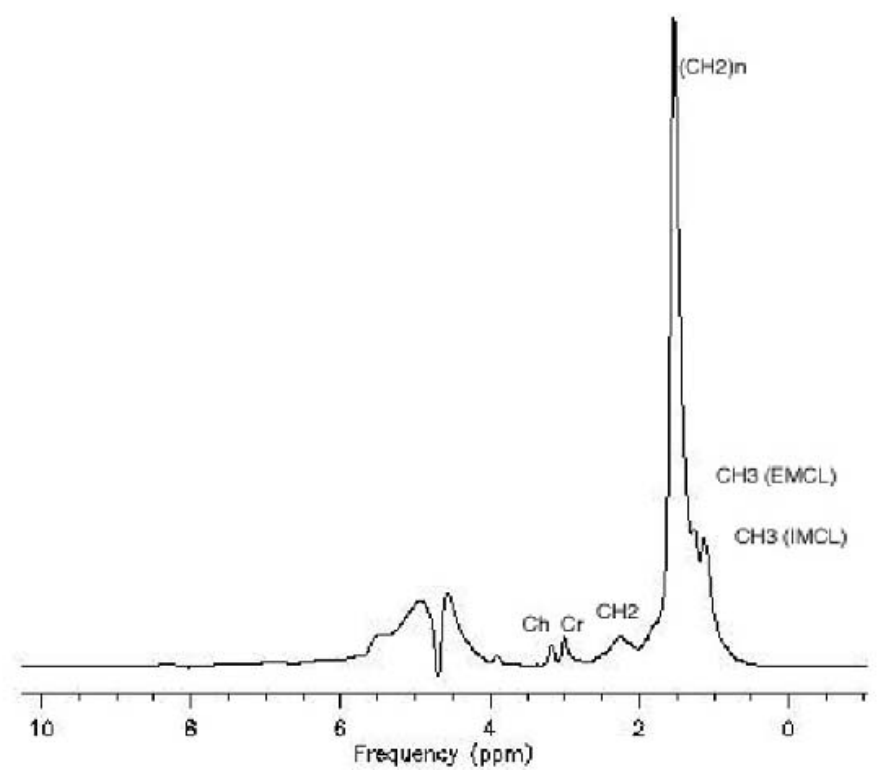

Figure 6.10: One-dimensional spectrum of the voxel on human calf muscle

The cross peaks of EMCL and IMCL are resolved very well, in the spectrum obtained using DQF-COSY. This is shown in Fig 6.11. The peaks $C 1$ and $C 1^{\prime}$ are due to EMCL and the peaks $C 2$ and $C 2^{\prime}$ are due to IMCL. 


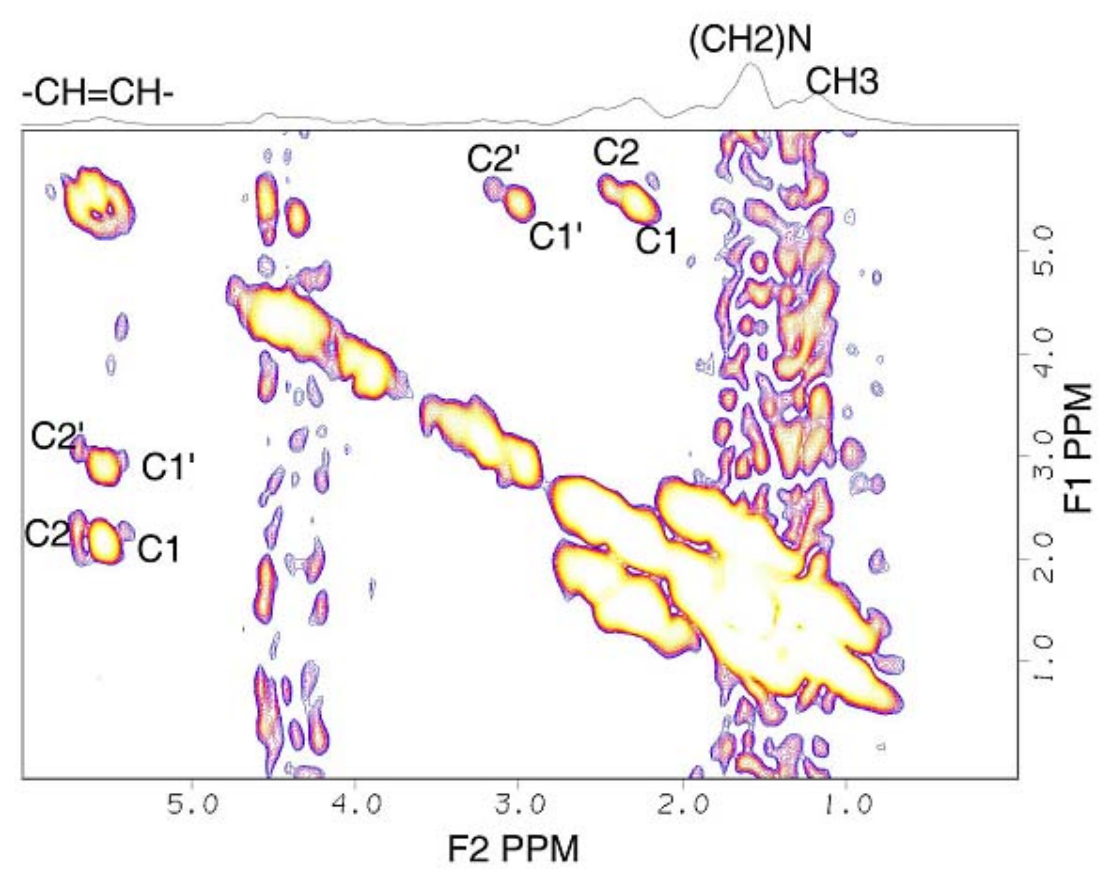

Figure 6.11: DQF-COSY spectrum of the voxel on human calf muscle 


\section{Chapter 7}

\section{Summary, Conclusion and Future Research}

In this thesis we have designed and developed a localized double-quantum-filtered correlated spectroscopy (DQF-COSY) pulse sequence. The volume localization and double quantum filtration have been achieved with three RF pulses. The slice selective gradients in the presence of the three $90^{\circ} \mathrm{RF}$ pulses in orthogonal directions select the voxel. The first two RF pulses prepare the multiple quantum coherences (MQC's). The first $90^{\circ} \mathrm{RF}$ pulse creates single quantum coherences, and they evolve under the chemical shifts, couplings and gradients. The second $90^{\circ} \mathrm{RF}$ pulse, distributes the magnetization from single quantum to higher quantum coherence. The multiple quantum coherences, evolve under the short time delay between the second and third $90^{\circ} \mathrm{RF}$ pulse. The third $90^{\circ} \mathrm{RF}$ pulse converts the multiple quantum coherences into single quantum coherences. Only double-quantum coherences are rephased as they pass through the double-quantum pathway. The other coherences are dephased. The double-quantum pathway is selected by the use of gradients in the ratio of 4:3:10.

2D spectra from phantom results show the suppression of single quantum coherences. Preliminary in vivo results on the calf muscle, shows the cross peaks of EMCL and IMCL. The DQF-COSY spectrum shows the signal that has been acquired only from double-quantum coherences. This results in considerable simplification of the 
spectrum when compared to that of L-COSY. The peaks of the diagonal peaks in the L-COSY spectrum are dispersive in nature and spread far into the spectrum. This is due to the in phase diagonal peak magnetization. The diagonal peaks are not dispersive in the DQF-COSY due to the anti phase magnetization. One of the limitation of the sequence is that the magnetization obtained is less that $50 \%$ of the magnetization detected by L-COSY due to the pathway selection process. The advantages offered by the DQF-COSY technique is that suppression of signals that cannot create double quantum coherences

Further research is required to understand the dynamics involved in doublequantum coherences. This will improve quantification strategies. We also observer long range coupling in DQF-COSY results. This might be due to the additional pathways involving exchange and zero-quantum coherences, which has to be explored. 


\section{References}

[1] General Electric Company. Signa Horizon LX Customer LX EPIC Tools User's Manual, 2 edition, 1997-1998.

[2] Robin A. de Graff. In vivo NMR spectroscopy. John Wiley \& Sons, 1998.

[3] H. C. Torrey E. M. Purcell and R. V. Pound. Resonance absorption by nuclear magnetic moments in a solid. Physical Review, 69:37-38, 1946.

[4] W. W. Hansen F. Bloch and M. E. Packard. Nuclear induction. Physical Review, 69:127, 1946.

[5] F. Bloch. Nuclear induction. Physical Review, 70:460-473, 1946.

[6] W. W. Hansen F. Bloch and M. Packard. The nuclear induction epxeriment. Physical Review, 70(474-485), 1946.

[7] W. G. Prcotor and F. C. Yu. The dependence of a nuclear magnetic resonance frequency upon chemical compound. Physical Reviews, 77:717, 1950.

[8] W. C. Dickinson. Dependence of the $\mathrm{F}^{19}$ nuclear resonance position on chemical compound. Physical Review, 77:736, 1950.

[9] R. R. Ernst and W. A. Anderson. Applications of fourier transform spectroscopy to magnetic resonance. Review of Scientific Instruments, 37:93-102, 1966.

[10] T. M. Shaw and R. H. Elsken. Nuclear magnetic resonance absorption in hygroscopic materials. Journal of Chemical Physics, 18:1113-1114, 1959.

[11] E. Odebald and G. Lindstrom. Some preliminary observations on the proton magnetic resonance in biological samples. Acta Radiol, 43:469-476, 1955.

[12] R. Damadian. Tumour detection by nulcear magnetic resonance. Science, 171:1151-1153, 1971.

[13] R. B. Moon and J. H. Richards. Determination of intracellular ph by ${ }^{31} \mathrm{P}$ magnetic resonance. Journal of Biological Chemistry, 248:7276-7278, 1973.

[14] D. G. Gadian G. K. Radda R. E. Richards D. I. Hoult, S. J. W. Busby and P. J. Seeley. Observations of tissue metabolites using ${ }^{31} \mathrm{P}$ nuclear magnetic resonanace. Nature, Lond, 252:285-287, 1974. 
[15] J. W. Prichard T. Jue C.C Hanstock, D. L. Rothman and R. G. Shulman. Spatially localized nmr spectra of metabolites in the human brain. Proc Natl Acad Sci USA, 85:1821-1825, 1988.

[16] M. L. Gyngell K. D. Merboldt W. Hanicke H. Braun, J. Frahm and R. Suter. Cerebral metabolism in man after acute stroke: new observation using localized proton nmr spectroscopy. Magnetic Resonance in Medicine, 9:126-131, 1989.

[17] J. Pan J. Hugg C. Palmer F. Gilliam E. Faught R. Kuzniecky, H. Hetherington and R. Morawetz. Proton spectroscopic imaging at 4.1 tesla in patients with malformations of cortical development and epilepsy. Neurology, 48:1018-1024, 1997.

[18] K. L. Behar D. L. Rothman, O. A. C Petroff and R. H. Mattson. Localized ${ }^{1} h$ nmr measurements of $\gamma$-aminobutyric acid in human brain in vivo. Proc Natl Acad Sci USA, 90:56625666, 1993.

[19] D. D Spencer K. L. Behar, D. L. Rothman and O. A. C Petroff. Analysis of macromolecule resonances in ${ }^{1} h \mathrm{nmr}$ spectra of human brain. Magnetic Resonance in Medicine, 32:294-302, 1994.

[20] Bd. B. Frederick J. R. Keltner, L. L. Wald and P. F. Renshaw. In vivo detection of gaba in human brain using a localized double-quantum filter technique. Magnetic Resonance in Medicine, 37:366-371, 1997.

[21] D. J. Meyerhoff M. A. Thomas, H. P. Hetherington and D. Twieg. Localized double quantum filtered nmr spectroscopy. Journal of Magnetic Resonance, B93:485-496, 1991.

[22] A. Wilman and P. Allen. In vivo nmr detection strategies for g-aminobutyric acid, utilizing proton spectroscopy and coherence-pathway filtering with gradients. Journal of Magnetic Resonance, B101:165171, 1993.

[23] J.A Sorenson L. N. Ryner and M. A. Thomas. 3d localized 2d nmr spectroscopy on an mri scanner. Journal of Magnetic Resonance, B107:126-137, 1995.

[24] J. A. Sorenson L. N. Ryner and M. A. Thomas. Localized $2 \mathrm{~d}$ j-resolved ${ }^{1} h \mathrm{mr}$ spectroscopy: strong coupling effects in vitro and in vivo. Magnetic Resonance in Imaging, 13:853-869, 1995.

[25] M. Mehta P. Turski M. A. Thomas, L. N. Ryner and J. A. Sorenson. Localized $2 \mathrm{~d}$ j-resolved ${ }^{1} h \mathrm{mr}$ spectroscopy of human brain tumors in vivo. Journal of Magnetic Resonance in Imaging, 6:453-459, 1996.

[26] N. Binesh P. Davanzo A. Kumar B. Siegel M. Frye J. Curran R. Lufkin P. Martin M. A. Thomas, K. Yue and Barry Guze. Localized two-dimensional shift correlated mr spectroscopy of human brain. Magnetic Resonance in Medicine, 46:58-67, 2001. 
[27] J. Jeener. Technical report, Ampere Summer School, Basko Polje, Yugoslavia, 1991.

[28] H. Subramanian J. S. Leigh M. Shinnar, S. Eleff. The synthesis of pulse sequences yielding arbitrary magnetization vectors. Magn. Reson. Med, 12:74-80, 1989.

[29] J. S. Leigh M. Shinnar, L. Bolinger. The use of finite impulse response filters in pulse design. Magn. Reson. Med, 12:81-87, 1989.

[30] J. S. Leigh M. Shinnar, L. Bolinger. The synthesis of soft pulses with a specified frequency response. Magn. Reson. Med., 12:88-92, 1989.

[31] J. S. Leigh M. Shinnar. The application of spinors to pulse synthesis and analysis. Magn. Reson. Med., 12:93-98, 1989.

[32] French patent 8610179, 1986.

[33] D. Nishimura J. Pauly, P. Le Roux and A. Macovski. Parameter relations for the shinnar-le roux selective excitation pulse design algorithm. IEEE Transactions on Medical Imaging, 10:53-65, 1991.

[34] A. Connelly R.J. Ordidge and J. A. B. Lohman. Image-selected in vivo spectroscopy (isis). Journal of Magnetic Resonance, 66:283-294, 1986.

[35] K-D. Merboldt J. Frahm and W. Hanicke. Localized proton spectroscopy using stimulated echoes. Journal of Magnetic Resonance, 70(488-492), 1986.

[36] E. L. Hahn. Spin echoes. Physics Reviews, 80:580-594, 1950.

[37] P. A. Bottomley. Selective volume method for performing localized nmr spectroscopy. U. S. patent 4480228, 1984.

[38] P. A. Bottomley. Spatial localization in nmr spectroscopy in vivo. Annals of the New York Academy of Sciences, 508:333-348, 1987.

[39] W. I. Jung. Localized double spin echo proton spectroscopy part i:basic concepts. Concepts in magnetic resonance, 8(1-17), 1996.

[40] W. I. Jung. Localized double spin echo proton spectroscopy part ii:weakly coupled homonuclear spin systems. Concepts in magnetic resonance, 8(77-103), 1996.

[41] Th. B. Foster P. A. Bottomley and R. D. Darrow. Depth-resolved surface coil spectroscopy (dress) for in vivo ${ }^{1} h,{ }^{3} 1 p$ and ${ }^{1} 3 c$ nmr. Journal of Magnetic Resonance, 59:338-342, 1981.

[42] E. Bartholdi W. P. Aue and R. R. Ernst. Two-dimensional spectroscopy. application to nuclear magnetic resonance. Journal of Chemical Physics, 64:2229-2246, 1976. 
[43] M. H. Levitt G. Bodenhausen O. W. Sorensen, G. W. Eich and R. R. Ernst. Product operator formalism for the description of nmr pulse experiments. Progr. NMR Spectrosc, 16:163-192, 1983.

[44] A. Wokaun. PhD thesis, 1978.

[45] A. Wokaun and R.R. Ernst. Chemical Physics Letters, pages 407-459, 1977.

[46] A. Wokaun A.A. Maudsley and R.R. Ernst. Chemical Physics Letters, pages 9-64, 1978.

[47] A. D. Bain. Journal of Magnetic Resonance, pages 407-459, 1984.

[48] H. Kogler G. Bodenhausen and R. R. Ernst. Journal of Magnetic Resonance, pages 370-428, 1984.

[49] D. P. Weitekamp. Advances in Magnetic Resonance, pages 111-122.

[50] A. F. Mehlkopf A. Bax, P.G. deJong and J. Smidt. Chemical Physics Letters, pages 567-636.

[51] M. H. Levitt C. J. R. Counsell and R. R. Ernst. Journal of Magnetic Resonance, pages 470-534, 1985.

[52] O. W. Sorensen U. Piantini and R. R. Ernst. Multiple quantum filters for elucidating nmr coupling networks. Journal of the American Chemical Society, 104:6800-6801, 1982.

[53] A. J. Shaka and R. Freeman. Simplification of nmr spectra by filtration through multiple quantum coherence. Journal of Magnetic Resonance, 51:169-173, 1983.

[54] Jean-Francois Dauphin Anthony A. Shaw, Christophe Salaun and Bernard Ancian. Artifact-free pfg-enhanced double-quantum-filtered cosy experiments. Journal of Magnetic Resonance, Series A 120:110-115, 1996.

[55] Ray Freeman. Spin Choreography. Oxford University Press, 1998.

[56] Ernest B. Cady. Clinical Magnetic Resonance Spectroscopy. Plenum Press, 1990.

[57] Wallace S. Brey, editor. Pulse Methods in 1D and 2D NMR. Academic Press Inc, 1987.

[58] Thomas C. Farrar and Edwin D. Becker. Pulse and Fourier Transform NMR. Academic Press, 1971.

[59] Ian R. Young and H Cecil Charles, editors. MR Spectroscopy: Clinical Applications and Techniques. Martin Dunitz, 1996.

[60] Frank A. Bovey. Nuclear Magnetic Resonance Spectroscopy. Academic Press, Inc, second edition, 1987. 
[61] NMR Spectroscopy: An Introduction. John Wiley \& Sons, 1980.

[62] Roger S. Macomber. A complete introduction to modern NMR Spectroscopy. John Wiley \& Sons, 1998.

[63] William R. Croasmun Robert M.K. Carlson. Two-Dimensional NMR Spectroscopy. VCH Publishers Inc, 1994.

[64] P. J. Hore. Nulcear Magnetic Resonance. II. Oxford Science Publications, 1995. 